\title{
AGE CONSTRAINTS FOR DEPOSITION AND SEDIMENTARY PROVENANCE OF ESPINHAÇO SUPERGROUP AND BAMBUÍ GROUP IN
}

\section{EASTERN SÃO FRANCISCO CRATON}

\author{
Matheus Kuchenbecker ${ }^{1,2}$, Humberto Luis Siqueira Reis ${ }^{1}$, Luiz Carlos da Silva ${ }^{4}$, Ricardo Diniz da \\ Costa르. Daniel Galvão Carnier Fragoso ${ }^{3}$, Luiz Guilherme Knauer², Ivo Antônio Dussin², Antônio \\ Carlos Pedrosa Soares ${ }^{2}$
}

\begin{abstract}
${ }^{1}$ Laboratório de Estudos Tectônicos/Núcleo de Geociências e Instituto de Ciência e Tecnologia, Universidade Federal dos Vales do Jequitinhonha e Mucuri, BR-367, Km 583, Alto da Jacuba, CEP 39100-000 Diamantina, MG, Brazil. E-mail: mk.geologia@gmail.com; ${ }^{2}$ Centro de Pesquisa Prof. Manoel Teixeira da Costa, Instituto de Geociências, Universidade Federal de Minas Gerais; ${ }^{3}$ PETROBRAS S.A.; ${ }^{4}$ CPRM - Serviço Geológico do Brasil
\end{abstract}

\begin{abstract}
The São Francisco Craton corresponds to an inner and stable portion of one of the plates involved in the assembly of Gondwanaland in late Neoproterozoic. To the east, the São Francisco Craton is limited by the Araçuaí-West Congo Orogen, which is composed, in its outer part, by the metasedimentary units of the Espinhaço Supergroup and Macaúbas Group. The cratonic adjacent area is mostly covered by the marine neoproterozoic units of the Bambuí Group, with few exposures of the Espinhaço Supergroup rocks. This paper aims to discuss the available geochronolgicl data and to present five new SHRIMP U-Pb dating on detrital zircons from rocks of the Bambuí Group and upper Espinhaço Supergroup at the eastern São Francisco Craton. The available dataset indicate that, within the São Francisco Craton, the upper portion of the Espinhaço Supergroup was deposited in the Pirapora Aulacogen through, in a period constrained between $1280 \mathrm{Ma}$ (younger zircon population) and $933 \mathrm{Ma}$ (age of the intrusive Pedro Lessa Mataigneous Suit). The cratonic basement highs (Sete Lagoas and Januária) seem to have been important source areas to the Mesoproterozoic units. The ages of the zircons found in the Serra de Santa Helena Formation suggest that its sediments may have come both from the Brasília Belt or the Araçuaí Orogen. On the other hand, zircons as young as $580 \mathrm{Ma}$, found in the Três Marias Formation sandstones indicate contribution from the Araçuaí Orogen. An important part of the sediment supply for the Espinhaço and Bambuí basins in the studied area could have come from older sedimentary units, as suggested by the great overlap in their detrital zircon age spectra. It demonstrates the remarkable polycyclic nature of the sedimentary processes who took place in the São Francisco Craton evolution
\end{abstract}

Keywords: Geochronology, São Francisco Craton, Pirapora Aulacogen, Bambuí Group, Espinhaço Supergroup

Resumo: PROVENIÊNCIA SEDIMENTAR E BALIZADORES DE IDADE DE DEPOSIÇÃO DAS ROCHAS DO SUPERGRUPO ESPINHAÇO E GRUPO BAMBUÍ NA PORÇÃO LESTE DO CRÁTON DO SÃO FRANCISCO. O Cráton do São Francisco corresponde à porção interna e estável de uma das placas envolvidas na aglutinação do supercontinente Gondwana, no fim do Neoproterozoico. Em sua borda leste o cráton é limitado pelo Orógeno Araçuaí-Congo Ocidental, que é composto, em sua porção externa, por rochas metassedimentares do Supergrupo Espinhaço e do Grupo Macaúbas. A área cratônica adjacente é, em grande parte, coberta por rochas do Grupo Bambuí, à exceção de grandes anticinórios, como o da Serra do Cabral, que exibem, em seu núcleo, rochas do Supergrupo Espinhaço. Este trabalho objetiva apresentar cinco novas datações U-Pb SHRIMP em zircões detríticos de rochas do Supergrupo Espinhaço e do Grupo Bambuí aflorantes na região da Serra do Cabral, integrando-as com os dados existentes. Em conjunto, os dados indicam que, na área cratônica, a porção superior do Supergrupo Espinhaço foi depositada entre $1280 \mathrm{Ma}$ (população mais jovem de zircões detríticos) e $933 \mathrm{Ma}$ (idade de rochas intrusivas básicas). Os altos de Sete Lagoas e Januária parecem ter atuado como importantes áreas-fonte para as unidades mesoproterozoicas. Os zircões encontrados em siltitos da Formação Serra de Santa Helena apresentam fontes possíveis na Faixa Brasília e no Orógeno Araçuaí, enquanto zircões com c.580 Ma, encontrados na Formação Três Marias, devem ter fonte exclusiva no Orógeno Araçuaí. Grande parte dos sedimentos que preencheram as bacias Espinhaço e Bambuí parece ter origem em unidades sedimentares mais antigas, como sugerido pela grande interseção entre seus espectros de idades de zircões detríticos, o que demonstra o notável caráter policíclico dos processos sedimentares.

Palavras-chave: Geocronologia, Cráton do São Francisco, Aulacógeno de Pirapora, Grupo Bambuí, Supergrupo Espinhaço

\section{INTRODUCTION}

The São Francisco Craton corresponds to an inner and stable portion of one of the plates involved in the assembly of Gondwanaland in late Neoproterozoic (Alkmim, 2004; Alkmim and MartinsNeto, 2012). To the west, the São Francisco Craton is limited by the Araçuaí-West Congo Orogen, which is composed, in its outer part, by the metasedimentary units of the Espinhaço Supergroup and Macaúbas Group. The cratonic adjacent area is mostly covered by the marine neoproterozoic units of the Bambuí Group.

Discussions concerning the depositional age and

doi: $10.18285 /$ geonomos.v23i2.708 the geotectonic setting of the Bambuí Group have been constant in the last decades. Babinski et al. (2007) obtained a Pb-Pb isochronic age of $740 \pm 22$ Ma for limestones of the Sete Lagoas Formation, pointing to a cryogenian age. However, in this same formation, zircon populations of c. $610 \mathrm{Ma}$ (Rodrigues, 2008, Pimentel et al. 2011) and $550 \mathrm{Ma}$ (Paula-Santos et al. 2012, Pimentel et al. 2012), as well as the occurrence of Cloudina sp. (Warren et al. 2014) indicating that the basin history could be more complex than previously thought. The Bambuí Basin has been considered a foreland basin linked to the building of the Brasília Belt (c. $630 \mathrm{Ma}$, Pimentel et al. 2011), which should have constituted its main source area (Martins-Neto et al. 2001, Martins-Neto 
and Alkmim 2001, Coelho et al. 2008, Kuchenbecker et al. 2013). Compounding the tectonic scenario, the collisional stage of the Araçuaí-West Congo orogen (starting at c. $580 \mathrm{Ma}$, Pedrosa-Soares et al. 2007) brings up new potential sources for the basin. This contribution would be recorded only in the upper part of the group (Martins-Neto and Alkmim 2001).

The evolution of the Espinhaço Supergroup has been also matter of debate. Athough is consensual that the Espinhaço basin starts its development in c. 1.75 Ga (Dussin \& Dussin 1995, Almeida-Abreu 1995, Knauer 2007) the historically proposed continuous sedimentation was questioned by Chemale Jr. et al. (2012): based on detrital zircon ages, the authors suggest a hiatus of c. 500 Ma within the supergroup. The uppermost part of the Espinhaço Supergroup (Conselheiro Mata Gr.) is represented by a shallow marine sequence (with rare dolomite lenses to the top), whose tectonic setting was also contested. Some have considered the unit as a record of a thermal SAG basin (e.g. Knauer 1999, Martins-Neto 1998, 2001), while others have proposed a foredeep context, related to west-vergent compressive tectonics (Almeida-Abreu et al. 2001).

In this controversial frame, studies focused on the dating of detrital zircons are becoming increasingly important, since the consolidation of a robust collection of data is crucial step to clarify these issues. With this in mind, this paper aims to discuss the available geochronolgical data and to present five new SHRIMP U-Pb dating on detrital zircons from rocks of the Bambuí Group and upper Espinhaço Supergroup at the eastern São Francisco Craton. The samples were collected in the context of the CPRM-UFMG mapping project, which mapped more than $21000 \mathrm{~km}^{2}$ in Minas Gerais during 2009/2010.

\section{GEOTECTONIC SETTING}

The São Francisco Craton (Almeida, 1977) and its african counterpart, the Congo Craton (Trompette, 1994), correspond to the stable parts of a Neoproterozoic palaeocontinent preserved from the Brasiliano-Pan African orogeny (Sial et al. 2009). In its southeastern limit, the Araçuaí-West Congo Orogen (AWCO, Fig. 1) represents a confined orogen evolved into an embayment (an inland-sea basin partially floored by oceanic crust) carved into the palaeocontinent (Pedrosa-Soares et al. 2001). At least three great aulacogens are recognized within the cratonic area, marking crustal unconformities which played an important role in the orogen evolution (Alkmim et al. 2006).
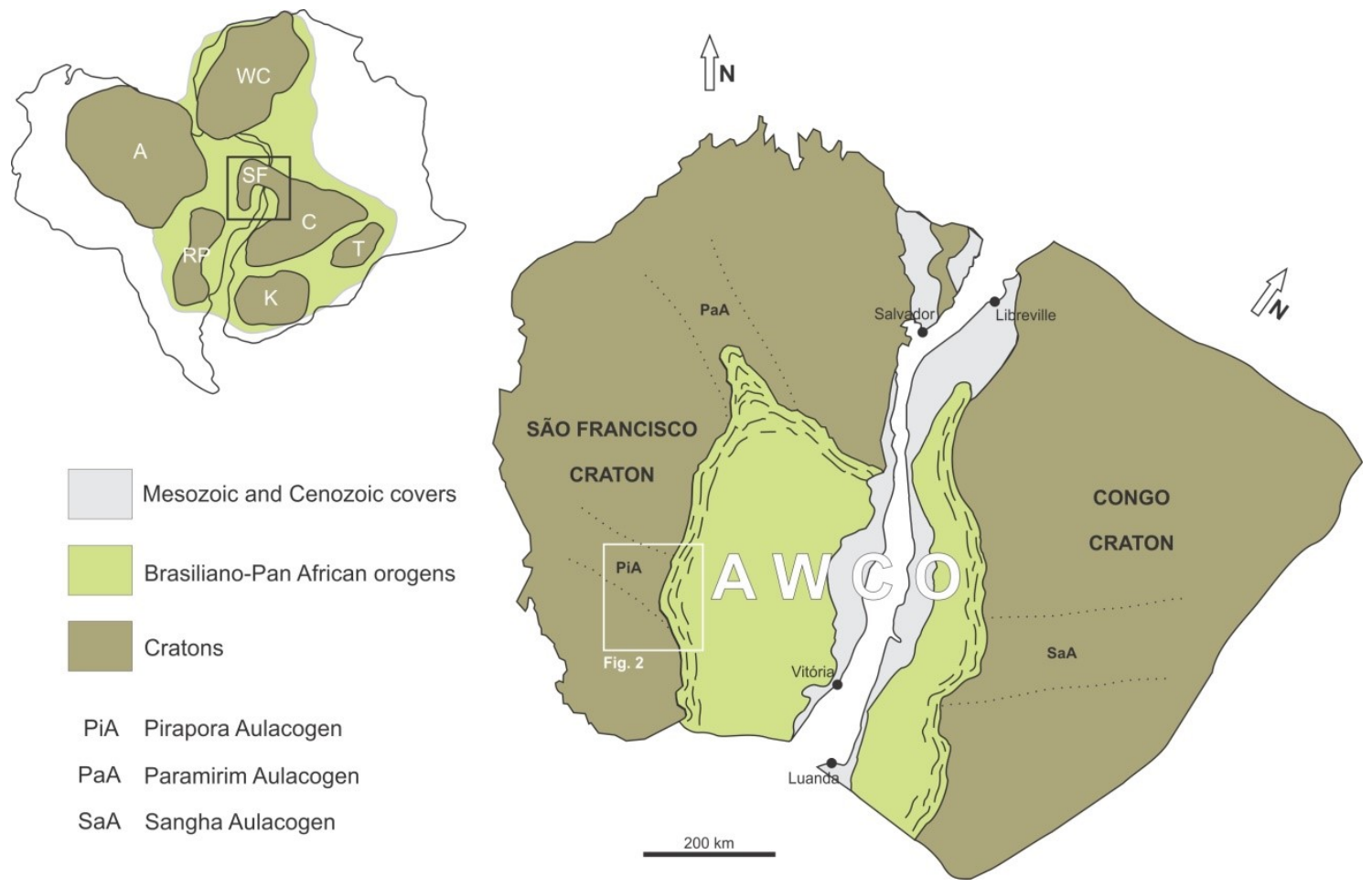

Figure 1. Geotectonic setting of the São Francisco-Congo Craton and the Araçuai West Congo Orogen, showing the positions of the aulacogens within the cratons. Based on Pedrosa-Soares et al. (2007). 


\section{REGIONAL GEOLOGY}

In the outer part of the $A O$, the Espinhaço Supergroup records the filling of a N-S-trending rift basin, with more than $2000 \mathrm{~m}$ thickness (Dussin and Dussin 1995). The rifting process is marked by bimodal magmatism, dated at c. $1.7 \mathrm{Ga}$ (Machado et al. 1989, Silva et al. 2002, Chemale Jr. et al. 2012). The basal units - São João da Chapada and SopaBrumadinho formations - are composed by continental quartzites and metaconglomerates (mainly fluvial, fan-deltaic and lacustrine deposits), besides metaigneous hematitic phyllite (Schöll and Fogaça 1979). Both formations are deposited in rift stage, under mechanical subsidence (Martins-Neto 1998). Recently, Chemale Jr. et al (2012) report zircons as young as c.1.2 Ga in the Sopa-Brumadinho Formation metaconglomerates, suggesting that most of the supergroup can be substantially younger.The Galho do Miguel Formation is a thick layer of pure quartzite deposited under aeolian influence, which records a quiet tectonic period, and the beginning of thermal subsidence (Martins-Neto 1998). In this phase, marine invasion is recorded in the Conselheiro Mata Group, the topmost unit of Espinhaço Supergroup. The Conselheiro Mata Group includes five formations: Santa Rita (metasiltstone), Córrego dos Borges (quartzite), Córrego da Bandeira (metasiltstone), Córrego Pereira (quartzite) and Rio Pardo Grande (metasiltstone, metadolomite). Both Galho do Miguel Formation and Conselheiro Mata Group occur within the São Francisco Craton, cropping out in anticline cores at the Cabral, Agua Fria and Bicudo ridges (Fig. 2).

The Bambui Group covers a large part of the SFC domain, and records a foreland basin related to the development of the orogenic Brasília Belt (MartinsNeto et al. 2001, Martins-Neto, 2009, Coelho et al. 2008). The basin includes basal diamictite-bearing units (Jequitaí and Carrancas formations), deposited under glacial influence (Rocha-Campos \& Hasui 1981, Kuchenbecker 2011). The overlain Bambuí Group represents a thick marine pelite-carbonate succession, arranged in three shallowing upward cycles. The group comprises five main units: (i) Sete Lagoas Formation, composed by limestones and dolomites (with local interbeded pelites), whose basal portion is interpreted as a cap carbonate (Vieira et al 2007, Babinski et al. 2007, Kuchenbecker 2011, Caxito et al. 2012, Paula-Santos et al. 2015); (ii) Serra de Santa Helena Formation, composed by shales and siltstones with rare interbeded limestones; (iii) Lagoa do Jacaré Formation, that shows reworked and oolitic limestones with pelitic layers; (iv) Serra da Saudade Formation, composed by siltstones an interbeded sandstones, with rares limestone lenses; and (v) Três Marias Formation, composed by arenites, arcoses and siltstones, with local occurrence of conglomerate, only in the east border of the basin.

In its west border, the AO is structured by $\mathrm{N}$ trending folds and thrusts. The main structural array of southern Serra do Espinhaço is controlled by regional thrusts/ductile inverse shear zones, invariably W-vergent (Knauer 2007, Knauer and Ebert 1997, Rolim 1992). To these features are associated a regional $\mathrm{N}$-axis folding system and several mesoscopic structures, such as a regional tectonic foliation, stretching lineation, crenulation cleavage, among others.

The trace of a major emergent thrust delineates the western edge of the AO (Alkmim et al. 2006). Along such structure, the Espinhaço Supergroup and Macaúbas Group rocks thrust upon the Bambuí Group rocks, and the deformation propagates inside the cratonic cover for a few kilometers. In the interaction zone between the deformational front and the Pirapora Aulacogen, the cratonic border is marked by the virtual lack of regional thrust zones, and the rocks shows only gentle folds (Fig. 2). Within the aulacogen through, such system is represented by regional sized bivergent folds like the Buenópilos sincline and the Cabral and Bicudo anticlines. Seismic profiles indicated that in depth these folds are related to blind faults that affected the cristaline basement (Reis et al. 2012, Hercos et al. 2008).

As well as the deformation, metamorphic grade decreases westward within the $A O$, ranging from granulite facies in the orogenic core, to low-grade metamorphic conditions at its borders. 


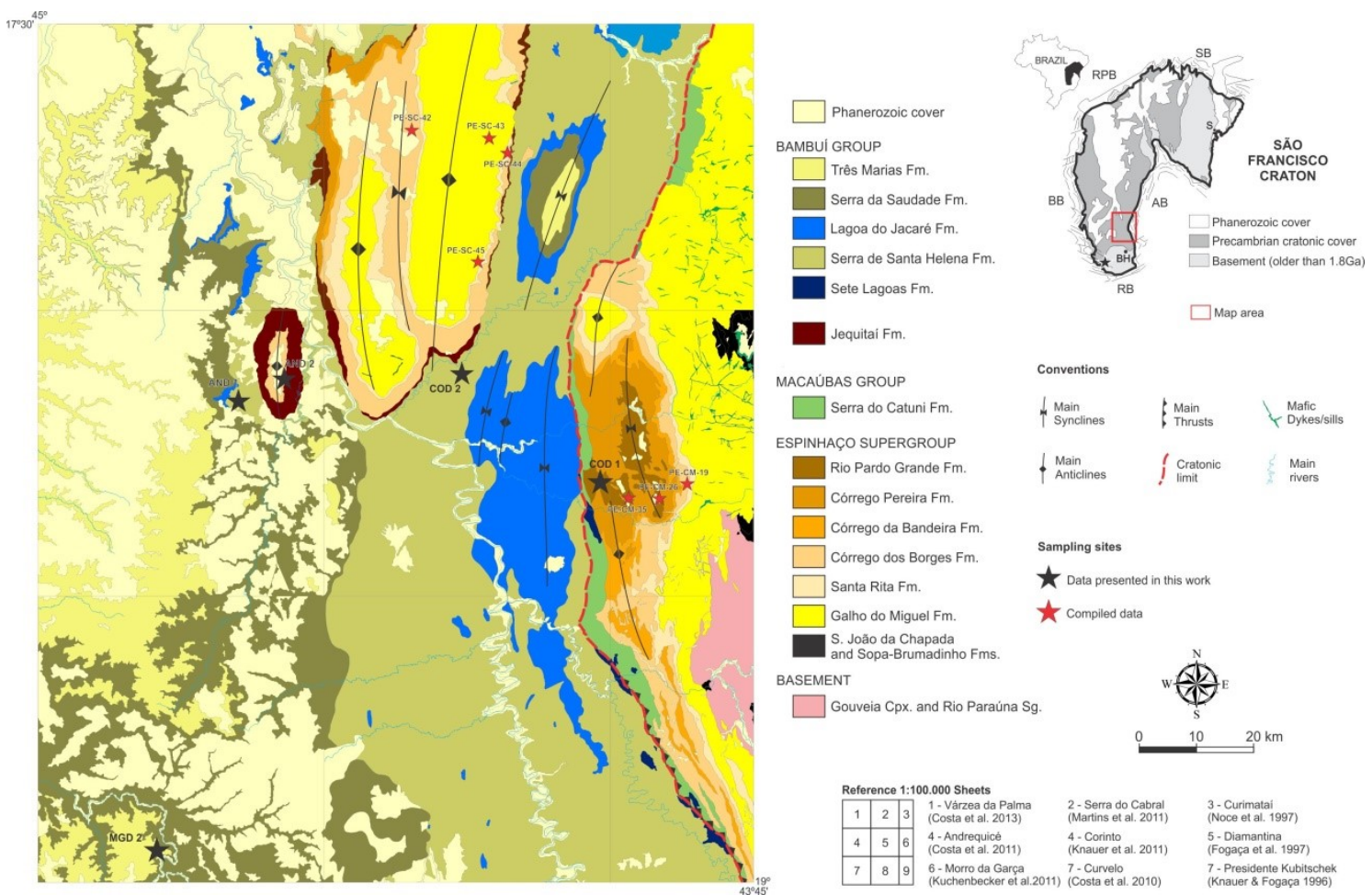

Figure 2. Simplified geological map from the studied area (see location in the Fig. 1), compiled from the official 1:100.000 sheet maps. Note that the main thrust zone which marks the cratonic limit in the south gives place to a regional fold system to the north, in interaction zone between the orogenic front and the Pirapora Aulacogen through.

\section{AVAILABLE DATA}

Seven U-Pb analyses in detrital zircons are available in the literature for the study area, and its results were compiled to improve the interpretation of our new data (Table 1). A brief description of these data is given below.

\subsection{Galho do Miguel Formation}

Two quartzite samples from Galho do Miguel Formation at the eastern flank of the Cabral ridge was analyzed by Lopes (2012). The samples show very similar main age peaks in 2000-2240 Ma. However, the sample PE-SC-45, collected to the south, has also an important peak in c. 2640-2760 $\mathrm{Ma}$, while the sample PE-SC-43, collected to the north, shows a peak in c. 1500-1700 Ma. This difference was interpreted by the author as the record of distinct sources providing sediments for different parts of the basin. The youngest grain reported has c. $1350 \mathrm{Ma}$, interpreted as the maximum depositional age of the Galho do Miguel Formation at the Cabral ridge.

\subsection{Santa Rita Formation}

A quartzite from Santa Rita Formation (sample PE-SC-44) was also collected in the eastern flank of Cabral ridge and analyzed by Lopes (2012). Thirtyfive zircons yielded ages ranging from 1480 to 3320 $\mathrm{Ma}$, with main age peaks at c. $1750 \mathrm{Ma}, 2100 \mathrm{Ma}$, $2270 \mathrm{Ma}$ and $2680 \mathrm{Ma}$, which was interpreted as the ages of the main sources of sediments.

\subsection{Córrego dos Borges Fm.}

Chemale Jr. et al. (2012) report analysis from a quartzite from this unit (sample PE-CM-19), collected in the western border of the Espinhaço ridge (locally known as Mineira ridge). The ages range between $1400 \mathrm{Ma}$ and $3200 \mathrm{Ma}$, with main peaks at 2080-2240 Ma and 1420-1560 Ma, and secondary peaks at $1720-1800 \mathrm{Ma}$ and $1940-1980$ $\mathrm{Ma}$. The youngest zircon was dated at $1395 \pm 30 \mathrm{Ma}$, interpreted as the maximum depositional age of the unit.

Lopes (2012) presents analysis from a quartzite from Córrego dos Borges Formation (sample PE-SC42) collected in the central portion of the Cabral ridge. The zircons yielded ages ranging 1480-3120 Ma and main peaks at 1750-1800 Ma, 2070-2170 Ma and 2620-2730 Ma. The author remarks the input of the $\mathrm{c} .1750 \mathrm{Ma}$ zircons on the basin, not reported by the subjacent units. The youngest zircon was dated at c. $1500 \mathrm{Ma}$.

\subsection{Córrego Pereira Formation}

Eighty zircon grains recovered from a quartzite of Córrego Pereira Formation (sample PE-CM-26) were dated by Chemale Jr. et al. (2012). The ages range between $1300 \mathrm{Ma}$ and $2200 \mathrm{Ma}$, with a large main peak at 1900-2025 Ma and a secondary peak at 1780-1850 Ma. The youngest zircon was dated at $1329 \pm 20$. 
Table 1. Summary of the available geochronological data from the Espinhaço Supergroup and Bambuí Group in the study area

\begin{tabular}{|c|c|c|c|c|c|c|c|}
\hline Sample & Unit & Rock & no of grains & Range (Ma) & Main Peaks (Ma) & Younger grain (Ma) & Reference \\
\hline PE-CM-35 & Rio Pardo Grande Fm. & Quartzite & 61 & $1500-2900$ & $1920-2020(62 \%)$ & $1514 \pm 12$ & Chemale Jr. et al. 2012 \\
\hline \multirow[t]{2}{*}{$P E-C M-26$} & Córrego Pereira Fm. & Quartzite & 80 & $1300-2200$ & $1780-1850(28 \%)$ & $1329 \pm 20$ & Chemale Jr. et al. 2012 \\
\hline & & & & & $1900-2025$ (67\%) & & \\
\hline \multirow[t]{3}{*}{$P E-S C-42$} & Córrego dos Borges Fm. & Quartzite & 69 & $1480-3120$ & $1750-1800(29 \%)$ & c. 1500 & Lopes 2012 \\
\hline & & & & & $2070-2170(23 \%)$ & & \\
\hline & & & & & $2620-2730(10 \%)$ & & \\
\hline \multirow[t]{4}{*}{$P E-C M-19$} & Córrego dos Borges Fm. & Quartzite & 69 & $1400-3200$ & $1420-1560(24 \%)$ & $1395 \pm 30$ & Chemale Jr. et al. 2012 \\
\hline & & & & & $1720-1800(15 \%)$ & & \\
\hline & & & & & $1940-1980(10 \%)$ & & \\
\hline & & & & & $2080-2240(28 \%)$ & & \\
\hline \multirow[t]{3}{*}{ PE-SC-44 } & Santa Rita Fm. & Quartzite & 35 & $1480-3320$ & $1650-1800(14 \%)$ & $1487 \pm 40$ & Lopes 2012 \\
\hline & & & & & $2040-2300(40 \%)$ & & \\
\hline & & & & & $2480-2680(20 \%)$ & & \\
\hline \multirow[t]{2}{*}{ PE-SC-43 } & Galho do Miguel Fm. & Quartzite & 74 & $1550-2950$ & $1500-1700(18 \%)$ & c. 1550 & Lopes 2012 \\
\hline & & & & & $2000-2240(65 \%)$ & & \\
\hline \multirow[t]{2}{*}{$P E-S C-45$} & Galho do Miguel Fm. & Quartzite & 90 & $1350-3350$ & $2000-2240(50 \%)$ & c. 1350 & Lopes 2012 \\
\hline & & & & & $2640-2760(27 \%)$ & & \\
\hline
\end{tabular}

\subsection{Rio Pardo Grande Formation}

A quartzite (sample PE-SC-35) from Rio Pardo Grande Formation was analyzed by Chemale Jr. et al. (2012). Sixty one grains were dated, and yielded ages between 1500 and $2900 \mathrm{Ma}$, with a large predominant peak at 1920-2020 Ma. The youngest zircon showed an age of $1514 \pm 12 \mathrm{Ma}$.

\section{NEW U-PB DATA}

\subsection{Analitical Procedures}

Heavy mineral concentrates were obtained by standard techniques, and zircon grains were handpicked under a binocular loupe. Zircon grains were mounted in a $2,5 \mathrm{~cm}$ diameter epoxy disk with standard RSES zircon crystals and sectioned approximately in half. The mount surface was then polished to expose the grain interiors and photographed to obtain cathodo-luminescence (CL) images. One spot was analyzed within each grain, preferably in its rim, and only the analyses that are less than $10 \%$ discordant were considered in discussions.

The $\mathrm{U}-\mathrm{Pb}$ isotopic analyses were performed by using Sensitive High Resolution Ion Microprobe (SHRIMP) equipment of Australian National University according to its conventional routines. Analytical methods and data treatment followed those described by Williams (1998) and Williams \& Meyer (1998). Temora (417 Ma; Black et al., 2003) standard zircon was used in SHRIMP analytical routines, and data reduction used the SQUID software (Ludwig, 2001), according to the procedures described in Chemale et al (2012). The histograms were obtained with the software Isoplot/Ex (Ludwig, 2003).

\subsection{Results}

Espinhaço Supergroup - Conselheiro Mata Group

Quartzite from Córrego dos Borges Fm. (AND 2 coordinates $\left.545739^{E} / 7996784^{N}\right)$

Located west of the Cabral Range, the Bicudo Range is a north-axis brachyanticline that exposes the Espinhaço Supergroup rocks in its core. The Córrego dos Borges Formation is there represented by a pale, fine- to medium-grained quartzite, often with plane-parallel stratification and riplle marks (Fig. 3A). The sample yielded small zircons, in general rounded and dirty looking. The U-Pb age spectrum obtained in 23 detrital zircon grains range between 1190 and $2125 \mathrm{Ma}$ (Table 2), mainly concentrated in intervals at 1.20-1.35 Ga (19\%), 1.51.6 Ga (24\%), 1.6-1.7 Ga (26\%), 1.9-2.1 (19\%, Figure 4). A single zircon grain showed an age of $1190 \pm 25$ $\mathrm{Ma}$, and three grains define the youngest age population at c.1280 Ma, interpreted as the maximum depositional age of the Córrego dos Borges Formation quartzites.

Quartzite from Córrego Pereira Fm. (COD 1 coordinates $\left.604211^{E} / 7976060^{N}\right)$

The sample was collected in the unpaved road that connects Rodeador to Conselheiro Mata. The rock is a grayish fine quartzite, showing rhythmic plane-parallel lamination marked by $\mathrm{Fe}_{2} \mathrm{O}_{3}$, dipping $30-40^{\circ}$ west (Fig. 3B). The rock yielded a lot of subhedral zircon grains, in dirty short prisms. The 33 detrital zircon grains analyzed yielded $\mathrm{U}-\mathrm{Pb}$ ages ranging from 1150 to $2450 \mathrm{Ma}$ (Table 3), but most of them fall into three age intervals of $1.20-1.30 \mathrm{Ga}$ $(18 \%), 1.35-1.45 \mathrm{Ga}(18 \%)$ and $1.8-2.0 \mathrm{Ga}(30 \%)$, suggesting that those were the ages of the most 
important sources of the sediments (Fig.4). The youngest zircon showed an age of $1150 \pm 19 \mathrm{Ma}$, and the youngest population fits at c.1230 Ma, which could be considered the maximum depositional age of the Córrego Pereira Formation metapelites.

\section{Bambuí Group}

Siltstone from Serra de Santa Helena Fm. (AND 1 - coordinates $537147^{E} / 7991897^{N}$ and COD 2 coordinates $\left.578184^{E} / 7997538^{N}\right)$

Two samples from Serra de Santa Helena Formation were collected at the study area: AND 1 is a brownish siltstone, collected west of the Bicudo ridge. The rock shows an outstanding plane-parallel lamination, and displays large amount of detrital mica. Gentle folds indicate weak deformation, consistent with that observed in the Bicudo Anticline (Fig. 3C). The zircons separated from this sample are very small and dirty looking, in subhedral to euhedral short prisms. The 47 zircons grains recovered from this sample yielded $\mathrm{U}-\mathrm{Pb}$ ages ranging from 620 to $2830 \mathrm{Ma}$ (Table 4), defining a largely predominant peak at $630-670 \mathrm{Ma}$ and secondary peaks at 0.7-0.9 Ga, 1.0-1.2 Ga, 1.8-2.0 Ga and 2.6-2.8 Ga (Fig. 4). The youngest zircon showed an ${ }^{206} \mathrm{~Pb} /{ }^{238} \mathrm{U}$ age of $622 \pm 7 \mathrm{Ma}$, which is interpreted as the maximum depositional age of this unit.

Sample COD 2 represents a pinkish silty mudstone, collected at the BR135 highway, near
Augusto de Lima city. The rock also displays planeparallel lamination and a large amount of detrital mica. Because of its fine grain size, only 12 zircon grains could be recovered from this sample, all dirty looking and sub-rounded. The zircon grains yielded $\mathrm{U}-\mathrm{Pb}$ ages ranging from 630 to $2850 \mathrm{Ma}$ (Table 5), showing main peaks at c. $630 \mathrm{Ma}$ e c. $2840 \mathrm{Ma}$.

Since the results obtained for the two Serra de Santa Helena Formation samples are quite similar, they are plotted together in the diagram (Fig 4.).

Sandstone from Três Marias Fm. (MGD 2 coordinates $\left.522780^{E} / 7904756^{N}\right)$

This sandstone was collected near the Paraopeba River, in the region of Retiro Baixo hydroelectric plant. The rock is a stratified silty sandstone (Fig. 3D), with decimetric layers of fine to medium sandstone, usually with a short amount of feldspar grains. The zircon grains separated from this sample were small, subhedral to euhedral (short prisms) or rounded. Forty-four zircons from this sample were dated and the ages range between 575 and $1920 \mathrm{Ma}$ (Table 6), distributed in a largely predominant peak at c. $650 \mathrm{Ma}$ and secondary peaks at c. $900 \mathrm{Ma}, 1500$ $\mathrm{Ma}$ and $1900 \mathrm{Ma}$ (Fig. 4). The youngest zircon showed an ${ }^{206} \mathrm{~Pb} /{ }^{238} \mathrm{U}$ age of $575 \pm 9 \mathrm{Ma}$, and we assume it as the maximum depositional age of the unit in this area.

Table 2. U-Pb SHRIMP data from detrital zircons of sample AND 2 (Córrego dos Borges Fm.) Notes: See Table 5

\begin{tabular}{|c|c|c|c|c|c|c|c|c|c|c|c|c|c|c|c|c|c|c|c|c|c|}
\hline \multirow{2}{*}{$\begin{array}{c}\text { Grain } \\
\text { Spot }\end{array}$} & \multirow{2}{*}{$\begin{array}{c}U \\
(p p m)\end{array}$} & \multirow{2}{*}{$\begin{array}{c}\text { Th } \\
\text { (ppm) }\end{array}$} & \multirow{2}{*}{$\mathrm{Th} / \mathrm{U}$} & \multirow{2}{*}{$\begin{array}{l}{ }^{206} \mathrm{~Pb}^{*} \\
\text { (ppm) }\end{array}$} & \multirow{2}{*}{$\begin{array}{c}{ }^{206} \mathrm{~Pb}^{\mathrm{c}} \\
\%\end{array}$} & \multicolumn{7}{|c|}{ Isotope ratios } & \multicolumn{6}{|c|}{ Age (Ma) } & \multirow{2}{*}{$\begin{array}{c}\text { Disc. } \\
\%\end{array}$} & \multirow{2}{*}{$\begin{array}{l}\text { Age } \\
\text { (Ma) }\end{array}$} & \multirow[b]{2}{*}{ \pm} \\
\hline & & & & & & $\begin{array}{r}207 \mathrm{~Pb}^{*} \\
\mathrm{I}^{235} \mathrm{U}\end{array}$ & $\pm(\%)$ & $\begin{array}{c}206 \mathrm{~Pb}^{*} / \\
{ }^{238} \mathrm{U}\end{array}$ & $\pm(\%)$ & $r$ & $\begin{array}{c}{ }^{207} \mathrm{~Pb}^{*} / \\
{ }^{206} \mathrm{~Pb}^{*}\end{array}$ & $\pm(\%)$ & $\begin{array}{c}{ }^{206} \mathrm{~Pb} / \\
{ }^{238} \mathrm{U}\end{array}$ & \pm & $\begin{array}{c}207 \\
\mathrm{~Pb} / \\
{ }^{235} \mathrm{U}\end{array}$ & \pm & $\begin{array}{l}207 \mathrm{~Pb} / \\
{ }^{206} \mathrm{~Pb}\end{array}$ & \pm & & & \\
\hline 2,1 & 250 & 190 & 0,78 & 61,39 & 0,111 & 4,175 & 2,86 & 0,285 & 2,02 & 0,70 & 0,106 & 2,03 & 1618 & 29 & 1669 & 48 & 1733 & 37 & 7 & 1629 & 53 \\
\hline 3,1 & 200 & 236 & 1,22 & 51,21 & 0,185 & 4,391 & 3,02 & 0,297 & 2,50 & 0,83 & 0,107 & 1,69 & 1677 & 37 & 1711 & 52 & 1752 & 31 & 4 & 1721 & 24 \\
\hline 6,1 & 214 & 131 & 0,63 & 56,30 & 0,000 & 4,422 & 2,50 & 0,306 & 1,99 & 0,80 & 0,105 & 1,51 & 1722 & 30 & 1716 & 43 & 1709 & 28 & -1 & 1715 & 20 \\
\hline 8,1 & 153 & 198 & 1,33 & 45,73 & 0,000 & 5,957 & 3,64 & 0,347 & 3,33 & 0,91 & 0,124 & 1,47 & 1921 & 55 & 1970 & 72 & 2021 & 26 & 5 & 2004 & 23 \\
\hline 9,1 & 94 & 35 & 0,38 & 21,25 & 0,000 & 3,534 & 4,28 & 0,262 & 2,97 & 0,69 & 0,098 & 3,09 & 1500 & 40 & 1535 & 66 & 1583 & 58 & 5 & 1526 & 33 \\
\hline 11,1 & 246 & 120 & 0,51 & 72,20 & 0,178 & 6,150 & 3,78 & 0,341 & 3,39 & 0,90 & 0,131 & 1,67 & 1892 & 56 & 1997 & 76 & 2108 & 29 & 10 & 2108 & 58 \\
\hline 12,1 & 116 & 54 & 0,48 & 28,92 & 0,000 & 4,220 & 3,18 & 0,290 & 2,36 & 0,74 & 0,106 & 2,14 & 1641 & 34 & 1678 & 53 & 1724 & 39 & 5 & 1676 & 26 \\
\hline 14,1 & 336 & 129 & 0,40 & 81,19 & 0,000 & 3,776 & 2,16 & 0,281 & 1,81 & 0,84 & 0,097 & 1,17 & 1596 & 26 & 1588 & 34 & 1576 & 22 & -1 & 1585 & 17 \\
\hline 15,1 & 223 & 120 & 0,56 & 68,57 & 0,098 & 6,518 & 2,14 & 0,358 & 1,86 & 0,87 & 0,132 & 1,06 & 1973 & 32 & 2048 & 44 & 2125 & 18 & 7 & 2125 & 37 \\
\hline 17,1 & 189 & 122 & 0,67 & 46,71 & 0,317 & 4,218 & 2,72 & 0,287 & 1,92 & 0,71 & 0,107 & 1,93 & 1625 & 28 & 1678 & 46 & 1744 & 35 & 7 & 1668 & 22 \\
\hline 18,1 & 90 & 71 & 0,82 & 26,67 & 0,000 & 5,242 & 3,04 & 0,346 & 2,59 & 0,85 & 0,110 & 1,60 & 1914 & 43 & 1859 & 57 & 1799 & 29 & -6 & 1836 & 24 \\
\hline 20,1 & 114 & 43 & 0,39 & 19,94 & 0,759 & 2,262 & 4,24 & 0,202 & 2,50 & 0,59 & 0,081 & 3,43 & 1184 & 27 & 1200 & 51 & 1230 & 67 & 4 & 1190 & 25 \\
\hline 24,1 & 262 & 210 & 0,83 & 85,55 & 0,096 & 6,569 & 2,21 & 0,379 & 1,96 & 0,88 & 0,126 & 1,03 & 2073 & 35 & 2055 & 45 & 2037 & 18 & -2 & 2045 & 16 \\
\hline 25,1 & 393 & 228 & 0,60 & 86,32 & 0,225 & 3,313 & 2,71 & 0,255 & 2,32 & 0,86 & 0,094 & 1,39 & 1464 & 30 & 1484 & 40 & 1513 & 26 & 3 & 1492 & 20 \\
\hline 30,1 & 277 & 46 & 0,17 & 51,99 & 0,512 & 2,509 & 4,47 & 0,217 & 3,24 & 0,73 & 0,084 & 3,08 & 1266 & 37 & 1275 & 57 & 1288 & 60 & 2 & 1272 & 32 \\
\hline 33,1 & 117 & 103 & 0,91 & 29,41 & 0,000 & 4,165 & 3,01 & 0,293 & 2,35 & 0,78 & 0,103 & 1,89 & 1656 & 34 & 1667 & 50 & 1681 & 35 & 1 & 1668 & 25 \\
\hline 36,1 & 196 & 178 & 0,94 & 54,61 & 0,000 & 4,919 & 2,71 & 0,325 & 2,33 & 0,86 & 0,110 & 1,37 & 1814 & 37 & 1805 & 49 & 1795 & 25 & -1 & 1801 & 21 \\
\hline 37,1 & 190 & 96 & 0,52 & 40,64 & 0,323 & 3,135 & 2,79 & 0,249 & 1,99 & 0,71 & 0,091 & 1,96 & 1431 & 25 & 1441 & 40 & 1456 & 37 & 2 & 1439 & 31 \\
\hline 38,1 & 441 & 292 & 0,68 & 85,22 & 0,149 & 2,818 & 3,28 & 0,225 & 2,84 & 0,87 & 0,091 & 1,64 & 1306 & 34 & 1360 & 45 & 1446 & 31 & 10 & 1381 & 23 \\
\hline 40,1 & 115 & 57 & 0,51 & 26,24 & 0,000 & 3,561 & 3,50 & 0,266 & 2,64 & 0,75 & 0,097 & 2,30 & 1519 & 36 & 1541 & 54 & 1571 & 43 & 3 & 1540 & 28 \\
\hline 41,1 & 33 & 32 & 1,01 & 8,75 & 0,000 & 4,980 & 4,90 & 0,308 & 3,43 & 0,70 & 0,117 & 3,50 & 1729 & 52 & 1816 & 89 & 1917 & 63 & 10 & 1800 & 42 \\
\hline 44,1 & 115 & 36 & 0,33 & 34,21 & 0,000 & 5,736 & 2,78 & 0,346 & 2,22 & 0,80 & 0,120 & 1,68 & 1915 & 37 & 1937 & 54 & 1960 & 30 & 2 & 1942 & 23 \\
\hline 46,1 & 206 & 120 & 0,60 & 38,17 & 0,324 & 2,539 & 3,03 & 0,215 & 1,91 & 0,63 & 0,086 & 2,35 & 1255 & 22 & 1190 & 36 & 1331 & 45 & 6 & 1268 & 20 \\
\hline
\end{tabular}


Table 3. U-Pb SHRIMP data from detrital zircons of sample COD 1 (Córrego Pereira Fm.) Notes: See Table 5.

\begin{tabular}{|c|c|c|c|c|c|c|c|c|c|c|c|c|c|c|c|c|c|c|c|c|c|}
\hline \multirow{2}{*}{$\begin{array}{c}\text { Grain } \\
\text { Spot }\end{array}$} & \multirow{2}{*}{$\underset{(\mathrm{ppm})}{\mathrm{U}}$} & \multirow{2}{*}{$\begin{array}{c}\text { Th } \\
\text { (ppm) }\end{array}$} & \multirow[b]{2}{*}{ /U } & \multirow{2}{*}{$\begin{array}{l}{ }^{206} \mathrm{~Pb}^{*} \\
\text { (ppm) }\end{array}$} & \multirow{2}{*}{${ }_{\%}^{206} \mathrm{~Pb}^{\mathrm{C}}$} & \multicolumn{7}{|c|}{ Isotope ratios } & \multicolumn{6}{|c|}{ Age (Ma) } & \multirow{2}{*}{$\begin{array}{c}\text { Disc. } \\
\%\end{array}$} & \multirow{2}{*}{$\begin{array}{l}\text { Age } \\
\text { (Ma) }\end{array}$} & \multirow[b]{2}{*}{ \pm} \\
\hline & & & & & & $\begin{array}{c}{ }^{207} \mathrm{~Pb}^{2} * / \\
{ }^{235} \mathrm{U}\end{array}$ & (\%) & $\mid \begin{array}{c}206 \\
\mathrm{~Pb}^{238} \mathrm{U}\end{array}$ & \pm & $r$ & $\begin{array}{l}{ }^{207} \mathrm{~Pb}^{*} / \\
{ }^{206} \mathrm{~Pb}^{*}\end{array}$ & (\%) & $\begin{array}{c}{ }^{206} \mathrm{~Pb} / \\
{ }^{238} \mathrm{U}\end{array}$ & \pm & $\begin{array}{l}\mathrm{Pb} / \\
{ }^{35} \mathrm{U}\end{array}$ & \pm & $\begin{array}{l}{ }^{207} \mathrm{~Pb} / \\
{ }^{206} \mathrm{~Pb}\end{array}$ & \pm & & & \\
\hline 1,1 & 47 & 32 & 23 & 9,13 & 000 & 2,803 & & 0,231 & & 65 & 0,088 & & 1342 & 24 & 1356 & 42 & 1379 & 45 & 3 & 350 & 21 \\
\hline 5,1 & 265 & 140 & 54 & 66,36 & 000 & 990 & 19 & 0,291 & 65 & 75 & 0,099 & 43 & 1646 & 24 & 1632 & 36 & 1614 & 27 & -2 & 632 & 18 \\
\hline 7,1 & 347 & 267 & 79 & 77,85 & 281 & 3,483 & 70 & 0,261 & 7 & 58 & 0,097 & 19 & 493 & 21 & 1523 & 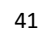 & 1566 & 41 & 5 & 507 & 19 \\
\hline 10,1 & 64 & 30 & 0,48 & 12,20 & 680 & 2,679 & 7,95 & 0,221 & 8 & 50 & 0,088 & 88 & 1289 & 46 & 1323 & 105 & 1378 & 132 & 6 & 1297 & 44 \\
\hline 11,1 & 78 & 44 & 59 & 26,13 & 000 & 6755 & 3,33 & 0,392 & 6 & 1 & 25 & 34 & 2133 & 13 & 2080 & 69 & 2028 & 42 & -5 & 078 & 30 \\
\hline 12,1 & 360 & 149 & 0,43 & 90,23 & 0,000 & 4,04 & 2,13 & 0,292 & 8 & 4 & 00 & 13 & 1651 & 3 & 1643 & 3 & 1632 & 27 & -1 & 1643 & 17 \\
\hline 13,1 & 130 & 107 & 0,86 & 39,03 & 000 & 5,62 & 2,86 & 0,351 & 1,89 & 6 & 6 & 15 & 937 & 2 & 1920 & 5 & 1901 & 39 & -2 & 23 & 24 \\
\hline 14,1 & 119 & 76 & 0,66 & 39,45 & 0,000 & 6,883 & 2,74 & 0,386 & 0 & 0,77 & 29 & 76 & 2102 & 38 & 2096 & 57 & 91 & 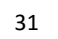 & -1 & 2095 & 24 \\
\hline 17,1 & 370 & 296 & 0,83 & 67,81 & 0,000 & 2,443 & 2,23 & 0,213 & 1,58 & 1 & 0,083 & 57 & 1247 & 18 & 1255 & 28 & 1270 & 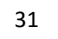 & 2 & 1252 & 16 \\
\hline 19,1 & 83 & 126 & 1,56 & 32,59 & 0,000 & 10,123 & 4,64 & 0,455 & 4,34 & 0,94 & 0,161 & 1,64 & 2418 & 87 & 2446 & 113 & 2470 & 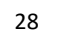 & 2 & 2465 & 26 \\
\hline 20,1 & 226 & 72 & 0,33 & 45,18 & 0,152 & 2,810 & 2,92 & 0,232 & 1,84 & 0,63 & 0,088 & 2,27 & 1347 & 22 & 2446 &  & 1377 & & 2 & 1353 & 20 \\
\hline 21,1 & 322 & 138 & 0,44 & 103,37 & 0,122 & 6,732 & 4,48 & 0,374 & 2,67 & 0,60 & 0,131 & 3,60 & 2047 & 47 & 2077 & 9 & 2107 & 63 & 3 & 2067 & 38 \\
\hline 22,1 & 219 & 156 & 0,74 & 60,25 & 0,000 & 4,712 & 1,86 & 0,321 & 1,65 & 0,88 & 0,107 & 0,87 & 1793 & 26 & 1769 & 3 & 1742 & 16 & -3 & 1756 & 14 \\
\hline 23,1 & 185 & 45 & 0,25 & 39,62 & 0,482 & 3,134 & 2,76 & 0,248 & 1,77 & 0,64 & 0,092 & 2,11 & 1427 & 23 & 1441 & 40 & 1461 & 4 & 2 & 1435 & 20 \\
\hline 24,1 & 158 & 107 & 0,70 & 49,85 & 0,000 & 6,022 & 2,42 & 0,366 & 1,9 & 0,79 & 19 & 1,48 & 2012 & 33 & 1979 & 48 & 1945 & 2 & -3 & 1971 & 21 \\
\hline 25,1 & 422 & 250 & 0,61 & 75,10 & 0,810 & 2,358 & 2,78 & 0,205 & 1,66 & 0,60 & 0,083 & 2,23 & 1204 & 18 & 1230 & 34 & 1276 & 44 & 6 & 1214 & 17 \\
\hline 26,1 & 222 & 177 & 0,83 & 58,36 & 0,201 & 4,090 & 2,55 & 0,306 & 1,78 & 0,70 & 0,097 & 1,82 & 1720 & 27 & 1652 & 42 & 1568 & 34 & -9 & 1568 & 67 \\
\hline 27,1 & 151 & 38 & 0,26 & 33,11 & 0,000 & 3,157 & 2,35 & 0,255 & 1,83 & 0,78 & 0,090 & 1,47 & 1464 & 24 & 1447 & 34 & 1422 & 28 & -3 & 1446 & 18 \\
\hline 28,1 & 190 & 170 & 0,93 & 56,12 & 0,096 & 5,475 & 2,37 & 0,344 & 1,93 & 0,81 & 0,115 & 1,38 & 1906 & 32 & 1897 & 45 & 1887 & 25 & -1 & 1894 & 20 \\
\hline 29,1 & 68 & 6 & 1,00 & 17,38 & 000 & 4,639 & 2,93 & 0,298 & 2,22 & 6 & 13 & 1,91 & 1684 & 33 & 1756 & 51 & 1844 & 35 & 10 & 1844 & 68 \\
\hline 32,1 & 249 & 105 & 0,44 & 68,70 &, 585 & 5,328 & 2,29 & 0,319 & 1,69 & 0,74 & 21 & 1,54 & 1787 & 26 & 1873 & 43 & 1970 & 21 & 10 & 1970 & 54 \\
\hline 36,1 & 269 & 0 & 0,42 & 57,75 & 345 & 3,092 & 2,40 & 0,249 & 1,75 & 0,73 & 0,090 & 1,64 & 1435 & 23 & 1431 & 34 & 1425 & 31 & -1 & 1431 & 18 \\
\hline 40,1 & 188 & 10 & 0,77 & 31,64 & 235 & 2,099 & 2,85 & 0,196 & 1,99 & 0 & 78 & 03 & 1152 & 21 & 1148 & 33 & 1142 & 40 & -1 & 150 & 19 \\
\hline 43,1 & 2 & 30 & 0,60 & 30 & 4 & & 12 & & 7 & 3 & 11 & 17 & 1665 & 26 & 1732 & 37 & & 21 & 9 & 13 & 42 \\
\hline 44,1 & 273 & 154 & 0 & 3 & 36 & & 2,53 & & 5 & 9 & 4 & 83 & 280 & 20 & $128 /$ & . & & 36 & 1 & 35 & 18 \\
\hline 46,1 & 34 & 132 & 1,0 & 9,48 & ,069 & 3,41 & 2,62 & 0,34 & 1,89 & $0, / 2$ & 0,113 & $1, c$ & 189 & 31 & 1887 & 43 & $18 / 9$ & 3 & -1 & 1887 & 22 \\
\hline 47,1 & $2 / 2$ & 134 & 0,58 & 2,07 & ,200 & 3,389 & 2,51 & 0,265 & 1,1 & 0,68 & 0,093 & 1,0 & 1515 & 23 & 1502 & 3 & 1484 & 35 & -2 & 1505 & 19 \\
\hline 49,1 & 115 & 88 & 0,7 & 3,12 & 168 & 5,266 & 2,51 & 0,335 & 1,95 & 0,78 & 0,114 & 1,3 & 1860 & 32 & 1863 & 41 & 1867 & 26 & 0 & 1864 & 21 \\
\hline 50,1 & 239 & 4 & 0,41 & 3,17 & ,380 & 2,334 & 2,99 & 0,209 & $1, / 5$ & 0,59 & 0,081 & 2,42 & $12 \angle 4$ & 20 & 1223 & 31 & 1219 & $4 c$ & 0 & 1224 & 18 \\
\hline 51,1 & 175 & 140 & 0,82 & 3,04 & ,196 & 2,663 & 2,53 & 0,219 & 1,84 & 0,73 & 0,088 & 1,73 & 1276 & 21 & 1318 & 33 & 1388 & 33 & 9 & 1388 & 65 \\
\hline 56,1 & 211 & 14 & 0,3 & 38,17 & 0,398 & 2,339 & 3,88 & 0,209 & 1,87 & 0,48 & 0,081 & 3,40 & 1225 & 21 & 1224 & 48 & 1222 & 67 & 0 & 1225 & 20 \\
\hline 64,1 & 96 & 81 & 0,87 & 27,73 & 0,000 & 5,085 & 2,56 & 0,336 & 2,04 & 0,80 & 0,110 & 1,54 & 1865 & 33 & 1834 & 47 & 1798 & 28 & -4 & 1826 & 21 \\
\hline$b, 1$ & 05 & & 0,60 & 26,02 & 0,108 & 5,446 & 2,64 & 0,341 & 2,06 & 0,78 & 0,116 & 1,65 & 1892 & 34 & 1892 & 50 & 1893 & 30 & 0 & 1892 & 22 \\
\hline
\end{tabular}
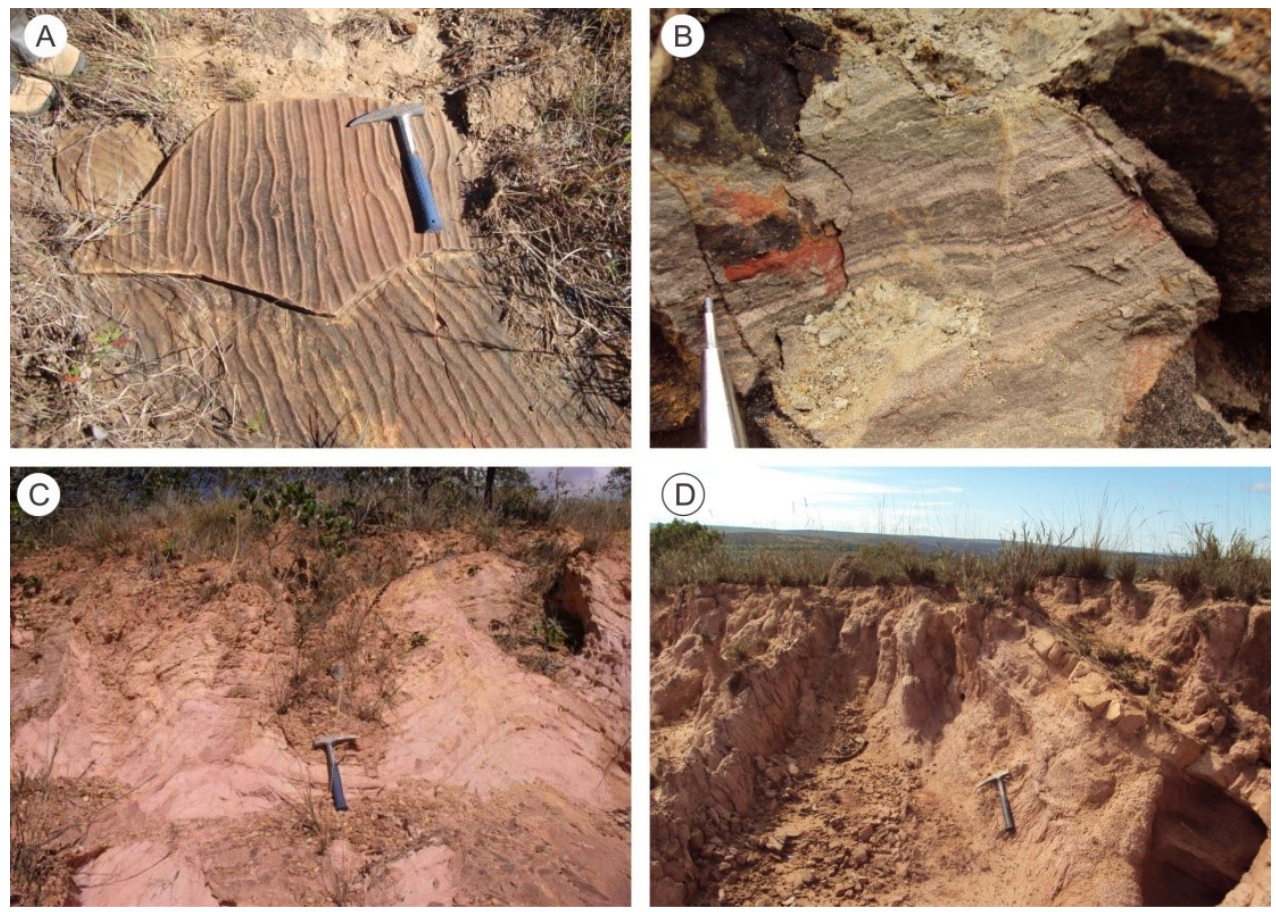

Figure 3. Field aspects of the studied units. (A) Córrego dos Borges quartzite, showing pronounced ripple marks; (B) Quartzite from Córrego Pereira Formation, with lamination marked by iron oxides; $(C)$ Laminated siltstone from Serra de Santa Helena formation, with metric sized gentle folds; (D) Stratified silty sandstone from the Três Marias Formation, showing a decimetric layer of pure sandstone. 
Table 4. U-Pb SHRIMP data from detrital zircons of sample AND 1 (Serra de Santa Helena Fm.). - Notes: See Table 5.

\begin{tabular}{|c|c|c|c|c|c|c|c|c|c|c|c|c|c|c|c|c|c|c|c|c|c|}
\hline \multirow{2}{*}{ Spot } & \multirow{2}{*}{$\underset{(p p m)}{U}$} & \multirow{2}{*}{$\begin{array}{c}\text { Th } \\
\text { (ppm) }\end{array}$} & \multirow[b]{2}{*}{ Th/U } & \multirow{2}{*}{$\begin{array}{l}{ }^{206} \mathrm{~Pb}^{*} \\
\text { (ppm) }\end{array}$} & \multirow{2}{*}{$\begin{array}{c}{ }^{206} \mathrm{Pbc} \\
\%\end{array}$} & \multicolumn{7}{|c|}{ Isotope ratios } & \multicolumn{6}{|c|}{ Age (Ma) } & \multirow{2}{*}{$\mid \begin{array}{c}\text { Disc } \\
. \%\end{array}$} & \multirow{2}{*}{$\begin{array}{l}\text { Age } \\
\text { (Ma) }\end{array}$} & \multirow[b]{2}{*}{ \pm} \\
\hline & & & & & & $\begin{array}{c}{ }^{207} \mathrm{~Pb}^{* 3} / \\
{ }^{235} \mathrm{U}\end{array}$ & $\pm(\%)$ & $\begin{array}{c}206 \mathrm{~Pb} * / \\
{ }^{238} \mathrm{U}\end{array}$ & $\pm(\%)$ & $r$ & $\begin{array}{c}{ }^{207} \mathrm{~Pb}^{*} / \\
{ }^{206} \mathrm{~Pb}^{*}\end{array}$ & $\pm(\%)$ & $\begin{array}{c}{ }^{206} \mathrm{~Pb} / \\
{ }^{238} \mathrm{U}\end{array}$ & \pm & $\begin{array}{c}{ }^{207} \mathrm{~Pb} / \\
{ }^{235} \mathrm{U}\end{array}$ & \pm & $\begin{array}{c}{ }^{207} \mathrm{~Pb} / \\
{ }^{206} \mathrm{~Pb}\end{array}$ & \pm & & & \\
\hline 1,1 & 68 & 169 & 2,56 & 13,6 & 0,591 & 2,709 & 3,45 & 0,231 & 1,62 & 0,53 & 0,085 & 3,04 & 1337 & 20 & 1331 & 46 & 1320 & 59 & -1 & 1336 & 19 \\
\hline 2,1 & 331 & 200 & 0,62 & 51,3 & 0,085 & 1,830 & 1,63 & 0,180 & 1,18 & 0,28 & 0,074 & 1,13 & 1069 & 12 & 1056 & 17 & 1029 & 23 & -4 & 1062 & 9 \\
\hline 3,1 & 505 & 598 & 1,22 & 46,7 & 0,898 & 0,900 & 2,82 & 0,107 & 1,14 & 0,60 & 0,061 & 2,58 & 653 & 7 & 652 & 18 & 648 & 55 & -1 & 653 & 7 \\
\hline 4,1 & 822 & 245 & 0,31 & 77,0 & 1,537 & 0,930 & 4,85 & 0,107 & 3,98 & 0,18 & 0,063 & 2,77 & 658 & 25 & 668 & 32 & 703 & 59 & 6 & 663 & 19 \\
\hline 5,1 & 637 & 335 & 0,54 & 60,3 & 0,970 & 0,925 & 4,46 & 0,109 & 1,13 & 0,75 & 0,061 & 4,31 & 668 & 7 & 665 & 30 & 655 & 93 & -2 & 669 & 6 \\
\hline 6,1 & 472 & 277 & 0,61 & 41,2 & 0,432 & 0,855 & 2,15 & 0,101 & 1,15 & 0,47 & 0,061 & 1,82 & 622 & 7 & 627 & 13 & 648 & 39 & 4 & 623 & 7 \\
\hline 8,1 & 664 & 444 & 0,69 & 58,7 & 0,105 & 0,860 & 1,50 & 0,103 & 1,15 & 0,24 & 0,061 & 0,97 & 631 & 7 & 630 & 9 & 626 & 21 & -1 & 631 & 6 \\
\hline 9,1 & 515 & 14 & 0,03 & 50,7 & 0,000 & 0,988 & 1,52 & 0,115 & 1,14 & 0,25 & 0,063 & 1,00 & 699 & 8 & 698 & 11 & 693 & 21 & -1 & 699 & 6 \\
\hline 11,1 & 297 & 298 & 1,04 & 44,7 & 0,000 & 1,781 & 1,55 & 0,175 & 1,19 & 0,23 & 0,074 & 0,99 & 1040 & 11 & 1039 & 16 & 1037 & 20 & 0 & 1039 & 8 \\
\hline 12,1 & 222 & 199 & 0,93 & 24,3 & 1,149 & 1,144 & 5,80 & 0,126 & 1,58 & 0,73 & 0,066 & 5,58 & 765 & 11 & 775 & 45 & 802 & 117 & 5 & 763 & 10 \\
\hline 15,1 & 54 & 59 & 1,13 & 5,3 & 0,112 & 0,982 & 3,93 & 0,114 & 1,89 & 0,52 & 0,062 & 3,45 & 697 & 12 & 694 & 27 & 686 & 74 & -2 & 697 & 12 \\
\hline 16,1 & 158 & 119 & 0,78 & 14,3 & 0,000 & 0,877 & 2,45 & 0,105 & 1,46 & 0,41 & 0,060 & 1,97 & 646 & 9 & 639 & 16 & 615 & 43 & -5 & 644 & 8 \\
\hline 17,1 & 93 & 67 & 0,75 & 13,8 & 0,000 & 1,753 & 2,36 & 0,173 & 1,52 & 0,36 & 0,074 & 1,80 & 1027 & 14 & 1028 & 24 & 1030 & 36 & 0 & 1028 & 12 \\
\hline 20,1 & 249 & 121 & 0,50 & 22,0 & 0,177 & 0,841 & 2,32 & 0,102 & 1,24 & 0,47 & 0,060 & 1,96 & 628 & 7 & 620 & 14 & 591 & 43 & -6 & 626 & 7 \\
\hline 21,1 & 623 & 185 & 0,31 & 274,4 & 0,197 & 14,178 & 1,19 & 0,512 & 1,13 & 0,05 & 0,201 & 0,37 & 2666 & 25 & 2762 & 33 & 2833 & 6 & 6 & 2833 & 52 \\
\hline 22,1 & 195 & 107 & 0,57 & 32,4 & 0,160 & 2,130 & 2,06 & 0,193 & 1,35 & 0,34 & 0,080 & 1,55 & 1137 & 14 & 1159 & 24 & 1200 & 31 & 5 & 1147 & 12 \\
\hline 26,1 & 322 & 177 & 0,57 & 31,2 & 0,158 & 0,952 & 2,05 & 0,113 & 1,22 & 0,40 & 0,061 & 1,65 & 688 & 8 & 679 & 14 & 652 & 35 & -5 & 685 & 7 \\
\hline 27,1 & 665 & 619 & 0,96 & 60,9 & 0,050 & 0,896 & 1,47 & 0,107 & 1,11 & 0,24 & 0,061 & 0,95 & 653 & 7 & 650 & 10 & 637 & 20 & -3 & 652 & 6 \\
\hline 28,1 & 102 & 106 & 1,08 & 12,2 & 0,000 & 1,300 & 2,69 & 0,140 & 1,56 & 0,42 & 0,067 & 2,18 & 843 & 12 & 846 & 23 & 853 & 45 & 1 & 844 & 11 \\
\hline 29,1 & 189 & 72 & 0,39 & 63,5 & 0,110 & 7,157 & 1,48 & 0,392 & 1,28 & 0,13 & 0,133 & 0,73 & 2130 & 23 & 2131 & 31 & 2133 & 13 & 0 & 2031 & 12 \\
\hline 30,1 & 342 & 246 & 0,74 & 99,4 & 0,497 & 5,894 & 1,49 & 0,337 & 1,22 & 0,18 & 0,127 & 0,85 & 1871 & 20 & 1960 & 29 & 2057 & 15 & 9 & 2057 & 61 \\
\hline 32,1 & 191 & 50 & 0,27 & 23,2 & 0,000 & 1,322 & 2,12 & 0,141 & 1,35 & 0,36 & 0,068 & 1,63 & 853 & 11 & 855 & 18 & 861 & 34 & 1 & 854 & 9 \\
\hline 33,1 & 127 & 96 & 0,78 & 11,4 & 1,046 & 0,856 & 6,23 & 0,103 & 1,54 & 0,75 & 0,060 & 6,04 & 634 & J & 628 & 39 & 607 & 131 & -4 & 635 & 8 \\
\hline 34,1 & 217 & 263 & 1,25 & 20,2 & 0,255 & 0,917 & 3,23 & 0,108 & 1,34 & 0,59 & 0,062 & 2,94 & 660 & 0 & 661 & 21 & 663 & 63 & 1 & 660 & 8 \\
\hline 35,1 & 676 & 553 & 0,85 & 248,7 & 0,460 & 9,819 & 1,23 & 0,427 & 1,10 & 0,11 & 0,167 & 0,56 & 2290 & 21 & 2418 & 30 & 2527 & 9 & 9 & 2527 & 52 \\
\hline 36,1 & 200 & 240 & 1,24 & 25,4 & 0,178 & 1,410 & 1,90 & 0,147 & 1,29 & 0,32 & 0,069 & 1,39 & 886 & 11 & 893 & 17 & 910 & 29 & 3 & 889 & 9 \\
\hline 37,1 & 46 & 52 & 1,19 & 13,6 & 0,326 & 5,527 & 2,70 & 0,346 & 1,92 & 0,29 & 0,116 & 1,90 & 1913 & 32 & 1905 & 51 & 1895 & 34 & -1 & 1907 & 21 \\
\hline 38,1 & 586 & 230 & 0,41 & 84,2 & 0,787 & 1,746 & 2,06 & 0,166 & 1,17 & 0,43 & 0,076 & 1,70 & 990 & 11 & 1026 & 21 & 1104 & 34 & 10 & 1104 & 74 \\
\hline 39,1 & 525 & 301 & 0,59 & 49,7 & 0,096 & 0,939 & 1,65 & 0,110 & 1,21 & 0,27 & 0,062 & 1,12 & 673 & 8 & 672 & 11 & 670 & 24 & 0 & 673 & 6 \\
\hline 40,1 & 255 & 279 & 1,13 & 39,0 & 0,000 & 1,825 & 1,66 & 0,178 & 1,24 & 0,25 & 0,074 & 1,10 & 1055 & 12 & 1054 & 18 & 1054 & 22 & 0 & 1055 & 9 \\
\hline 41,1 & 584 & 263 & 0,46 & 54,7 & 0,071 & 0,936 & 1,56 & 0,109 & 1,13 & 0,27 & 0,062 & 1,07 & 666 & 7 & 671 & 10 & 688 & 23 & 3 & 668 & 6 \\
\hline 42,1 & 163 & 62 & 0,39 & 14,9 & 0,105 & 0,897 & 2,50 & 0,106 & 1,38 & 0,45 & 0,061 & 2,08 & 651 & 9 & 650 & 16 & 645 & 45 & -1 & 651 & 8 \\
\hline 43,1 & 154 & 158 & 1,06 & 44,7 & 0,181 & 5,293 & 1,71 & 0,337 & 1,41 & 0,18 & 0,114 & 0,97 & 1874 & 23 & 1868 & 32 & 1861 & 17 & -1 & 1869 & 13 \\
\hline 45,1 & 166 & 154 & 0,96 & 46,3 & 0,153 & 4,939 & 1,67 & 0,324 & 1,39 & 0,17 & 0,110 & 0,93 & 1811 & 22 & 1809 & 30 & 1807 & 17 & 0 & 1809 & 13 \\
\hline 47,1 & 100 & 193 & 2,00 & 18,5 & 0,897 & 2,395 & 3,87 & 0,214 & 1,60 & 0,59 & 0,081 & 3,52 & 1251 & 18 & 1241 & 48 & 1223 & 69 & -2 & 1251 & 18 \\
\hline 49,1 & 90 & 101 & 1,16 & 15,8 & 0,309 & 2,205 & 2,79 & 0,204 & 1,59 & 0,43 & 0,078 & 2,29 & 1198 & 17 & 1183 & 33 & 1154 & 45 & -4 & 1192 & 15 \\
\hline 50,1 & 192 & 312 & 1,67 & 49,0 & 0,089 & 4,655 & 1,58 & 0,296 & 1,28 & 0,19 & 0,114 & 0,93 & 1671 & 19 & 1759 & 28 & 1865 & 17 & 10 & 1865 & 65 \\
\hline
\end{tabular}

Table 5. U-Pb SHRIMP data from detrital zircons of sample COD 2 (Serra de Santa Helena Fm.)

\begin{tabular}{|c|c|c|c|c|c|c|c|c|c|c|c|c|c|c|c|c|c|c|c|c|c|}
\hline \multirow[b]{2}{*}{ Spot } & \multirow{2}{*}{$\underset{(p p m)}{U}$} & \multirow{2}{*}{$\begin{array}{c}\text { Th } \\
\text { (ppm) }\end{array}$} & \multirow[b]{2}{*}{ Th/U } & \multirow{2}{*}{$\begin{array}{l}{ }^{206} \mathrm{~Pb}^{*} \\
\text { (ppm) }\end{array}$} & \multirow{2}{*}{$\begin{array}{c}{ }^{206} \mathrm{Pbc} \\
\%\end{array}$} & \multicolumn{7}{|c|}{ Isotope ratios } & \multicolumn{6}{|c|}{ Age (Ma) } & \multirow{2}{*}{$\begin{array}{c}\text { Disc. } \\
\%\end{array}$} & \multirow{2}{*}{$\begin{array}{l}\text { Age } \\
\text { (Ma) }\end{array}$} & \multirow[b]{2}{*}{ \pm} \\
\hline & & & & & & \begin{tabular}{|l|}
${ }^{207} \mathrm{~Pb}^{*}$ \\
${ }^{235} \mathrm{U}$
\end{tabular} & $\pm(\%)$ & $\begin{array}{c}{ }^{206} \mathrm{~Pb}^{*} * / \\
{ }^{238} \mathrm{U}\end{array}$ & $\pm(\%)$ & $r$ & $\begin{array}{c}{ }^{207} \mathrm{~Pb}^{*} / \\
{ }^{206} \mathrm{~Pb}^{*}\end{array}$ & $\pm(\%)$ & \begin{tabular}{|c|}
${ }^{206} \mathrm{~Pb} /$ \\
${ }^{238} \mathrm{U}$
\end{tabular} & \pm & $\begin{array}{c}{ }^{207} \mathrm{~Pb} / \\
{ }^{235} \mathrm{U}\end{array}$ & \pm & $\begin{array}{c}{ }^{207} \mathrm{~Pb} / \\
{ }^{206} \mathrm{~Pb}\end{array}$ & \pm & & & \\
\hline 2,1 & 246 & 299 & 1,25 & 117,78 & 0,170 & 15,474 & 1,22 & 0,556 & 1,16 & 0,95 & 0,202 & 0,39 & 2850 & 27 & 2845 & 35 & 2842 & 6 & 0 & 2842 & 7 \\
\hline 3,1 & 443 & 200 & 0,47 & 58,85 & 0,535 & 1,453 & 1,93 & 0,154 & 1,12 & 0,58 & 0,069 & 1,57 & 921 & 10 & 911 & 18 & 887 & 33 & -4 & 918 & 9 \\
\hline 4,1 & 561 & 50 & 0,09 & 165,53 & 0,411 & 5,656 & 1,20 & 0,342 & 1,08 & 0,90 & 0,120 & 0,53 & 1896 & 18 & 1925 & 23 & 1956 & 9 & 3 & 1956 & 19 \\
\hline 7,1 & 178 & 78 & 0,45 & 58,36 & 0,274 & 6,819 & 1,41 & 0,380 & 1,21 & 0,86 & 0,130 & 0,72 & 2075 & 21 & 2088 & 29 & 2101 & 13 & 1 & 2094 & 11 \\
\hline 8,1 & 2672 & 2384 & 0,92 & 236,03 & 0,124 & 0,875 & 1,23 & 0,103 & 1,04 & 0,84 & 0,062 & 0,66 & 630 & 6 & 638 & 8 & 667 & 14 & 6 & 667 & 28 \\
\hline 9,1 & 1322 & 812 & 0,63 & 116,19 & 0,115 & 0,860 & 1,26 & 0,102 & 1,05 & 0,83 & 0,061 & 0,69 & 627 & 6 & 630 & 8 & 640 & 15 & 2 & 629 & 6 \\
\hline 11,1 & 178 & 60 & 0,35 & 19,41 & 0,498 & 1,130 & 2,91 & 0,126 & 1,30 & 0,45 & 0,065 & 2,60 & 767 & 9 & 768 & 22 & 769 & 55 & 0 & 768 & 9 \\
\hline 13,1 & 103 & 62 & 0,63 & 45,54 & 0,145 & 13,020 & 1,53 & 0,515 & 1,39 & 0,91 & 0,183 & 0,63 & 2677 & 31 & 2681 & 41 & 2684 & 10 & 0 & 2683 & 10 \\
\hline 14,1 & 149 & 111 & 0,77 & 27,44 & 0,152 & 2,340 & 1,91 & 0,213 & 1,26 & 0,66 & 0,080 & 1,44 & 1247 & 14 & 1225 & 23 & 1185 & 28 & -5 & 1185 & 56 \\
\hline
\end{tabular}

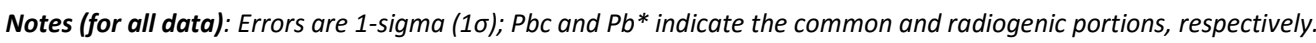
Error in Standard calibration was 0.50 and $0.36 \%$ (not included in above errors).

(1) Common Pb corrected using measured 204Pb. Isotopic ratios errors in \%.

$\mathrm{All} \mathrm{Pb}$ in ratios are radiogenic component. Most are corrected for 204Pb and some for 208Pb (metamorphic, Th-poor grains or rims). disc. $=$ discordance as $100-100\{t[206 \mathrm{~Pb} / 238 \mathrm{U}] / \mathrm{t}[207 \mathrm{~Pb} / 206 \mathrm{~Pb}]\}$

Uncertainties are $1 \sigma$. 
Table 6. U-Pb SHRIMP data from detrital zircons of sample MGD 2 (Três Marias Fm.) Notes: See Table 5.

\begin{tabular}{|c|c|c|c|c|c|c|c|c|c|c|c|c|c|c|c|c|c|c|c|c|c|}
\hline \multirow[b]{2}{*}{ Spot } & \multirow{2}{*}{$\underset{(p p m)}{U}$} & \multirow{2}{*}{$\begin{array}{c}\text { Th } \\
\text { (ppm) }\end{array}$} & \multirow{2}{*}{ Th/U } & \multirow{2}{*}{$\begin{array}{l}{ }^{206} \mathrm{~Pb}^{*} \\
\text { (ppm) }\end{array}$} & \multirow{2}{*}{$\begin{array}{c}{ }^{206} \mathrm{Pbc} \\
\%\end{array}$} & \multicolumn{7}{|c|}{ Isotope ratios } & \multicolumn{6}{|c|}{ Age (Ma) } & \multirow{2}{*}{$\begin{array}{c}\text { Disc. } \\
\%\end{array}$} & \multirow{2}{*}{$\begin{array}{l}\text { Age } \\
\text { (Ma) }\end{array}$} & \multirow[b]{2}{*}{ \pm} \\
\hline & & & & & & $\begin{array}{c}{ }^{207} \mathrm{~Pb}^{*} / \\
{ }^{235} \mathrm{U}\end{array}$ & $\pm(\%)$ & $\begin{array}{c}{ }^{206} \mathrm{~Pb}^{*} / \\
{ }^{238} \mathrm{U}\end{array}$ & $\pm(\%)$ & $r$ & $\begin{array}{c}207 \mathrm{~Pb}^{*} / \\
{ }^{206} \mathrm{~Pb}^{*}\end{array}$ & $\pm(\%)$ & $\begin{array}{c}{ }^{206} \mathrm{~Pb} / \\
{ }^{238} \mathrm{U}\end{array}$ & \pm & $\begin{array}{c}{ }^{207} \mathrm{~Pb} / \\
{ }^{235} \mathrm{U}\end{array}$ & \pm & $\begin{array}{c}207 \mathrm{~Pb} / \\
{ }^{206} \mathrm{~Pb}\end{array}$ & \pm & & & \\
\hline 1,1 & 50 & 401 & 8,37 & 14,52 & 0,108 & 5,446 & 2,64 & 0,341 & 2,06 & 0,78 & 0,116 & 1,65 & 1892 & 34 & 1892 & 50 & 1893 & 30 & 0 & 1892 & 44 \\
\hline 3,1 & 497 & 298 & 0,62 & 66,05 & 0,017 & 1,447 & 3,00 & 0,155 & 1,88 & 0,63 & 0,068 & 2,34 & 928 & 16 & 909 & 27 & 862 & 48 & -8 & 921 & 30 \\
\hline 4,1 & 90 & 54 & 0,63 & 10,25 & 0,922 & 1,224 & 10,08 & 0,132 & 2,44 & 0,24 & 0,067 & 9,78 & 798 & 18 & 812 & 82 & 849 & 203 & 6 & 798 & 36 \\
\hline 5,1 & 78 & 60 & 0,79 & 10,74 & 0,000 & 1,577 & 3,77 & 0,160 & 2,25 & 0,60 & 0,071 & 3,03 & 958 & 20 & 961 & 36 & 970 & 62 & 1 & 959 & 38 \\
\hline 6,1 & 383 & 196 & 0,53 & 60,91 & 0,472 & 1,886 & 3,03 & 0,184 & 1,72 & 0,57 & 0,074 & 2,49 & 1090 & 17 & 1076 & 33 & 1048 & 50 & -4 & 1086 & 32 \\
\hline 7,1 & 328 & 336 & 1,06 & 34,88 & 0,220 & 1,105 & 3,50 & 0,124 & 1,82 & 0,52 & 0,065 & 2,98 & 752 & 13 & 756 & 26 & 768 & 63 & 2 & 752 & 26 \\
\hline 8,1 & 1025 & 1332 & 1,34 & 82,47 & 0,362 & 0,763 & 2,46 & 0,093 & 1,55 & 0,63 & 0,059 & 1,91 & 575 & 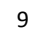 & 576 & 14 & 579 & 42 & 1 & 575 & 17 \\
\hline 9,1 & 607 & 162 & 0,28 & 68,65 & 0,434 & 1,167 & 3,63 & 0,131 & 1,80 & 0,50 & 0,065 & 3,15 & 794 & 13 & 785 & 28 & 760 & 66 & -4 & 793 & 26 \\
\hline 11,1 & 468 & 128 & 0,28 & 117,24 & 0,000 & 3,969 & 2,09 & 0,292 & 1,77 & 0,84 & 0,099 & 1,12 & 1651 & 26 & 1628 & 34 & 1599 & 21 & -3 & 1620 & 33 \\
\hline 12,1 & 123 & 134 & 1,13 & 35,83 & 0,482 & 5,680 & 3,04 & 0,339 & 2,05 & 0,68 & 0,122 & 2,24 & 1881 & 33 & 1928 & 59 & 1980 & 40 & 5 & 1920 & 52 \\
\hline 13,1 & 1711 & 474 & 0,29 & 155,02 & 0,064 & 0,892 & 1,79 & 0,105 & 1,54 & 0,86 & 0,061 & 0,91 & 646 &  & 648 & 12 & 653 & 20 & 1 & 647 & 17 \\
\hline 14,1 & 283 & 180 & 0,66 & 85,26 & 0,895 & 5,549 & 2,81 & 0,347 & 1,77 & 0,63 & 0,116 & 2,18 & 1922 & 29 & 1908 & 54 & 1893 & 39 & -2 & 1912 & 47 \\
\hline 15,1 & 3126 & 1092 & 0,36 & 293,56 & 0,230 & 0,923 & 1,76 & 0,109 & 1,50 & 0,85 & 0,061 & 0,93 & 667 & 9 & 664 & 12 & 652 & 20 & -2 & 664 & 17 \\
\hline 18,1 & 347 & 159 & 0,47 & 31,20 & 0,327 & 0,868 & 2,88 & 0,104 & 1,76 & 0,61 & 0,060 & 2,28 & 639 & 11 & 634 & 18 & 618 & 49 & -3 & 638 & 21 \\
\hline 20,1 & 567 & 581 & 1,06 & 62,07 & 0,283 & 1,141 & 2,65 & 0,127 & 1,66 & 0,63 & 0,065 & 2,07 & 771 & 12 & 773 & 20 & 778 & 43 & 1 & 771 & 23 \\
\hline 21,1 & 476 & 880 & 1,91 & 61,22 & 0,391 & 1,458 & 2,87 & 0,149 & 1,64 & 0,57 & 0,071 & 2,36 & 896 & 14 & 913 & 26 & 956 & 48 & 6 & 900 & 27 \\
\hline 22,1 & 602 & 553 & 0,95 & 48,44 & 0,000 & 0,768 & 2,25 & 0,094 & 1,62 & 0,72 & 0,059 & 1,57 & 577 & 9 & 579 & 13 & 585 & 34 & 1 & 578 & 17 \\
\hline 23,1 & 666 & 321 & 0,50 & 63,22 & 0,092 & 0,926 & 2,40 & 0,110 & 1,69 & 0,71 & 0,061 & 1,70 & 675 & 11 & 666 & 16 & 633 & 37 & -7 & 672 & 21 \\
\hline 24,1 & 512 & 417 & 0,84 & 46,36 & 0,328 & 0,875 & 3,05 & 0,105 & 1,66 & 0,55 & 0,060 & 2,56 & 644 & 10 & 638 & 19 & 617 & 55 & -4 & 643 & 20 \\
\hline 25,1 & 190 & 116 & 0,63 & 28,75 & 0,000 & 1,788 & 2,67 & 0,176 & 1,88 & 0,70 & 0,074 & 1,90 & 1044 & 18 & 1041 & 28 & 1035 & 38 & -1 & 1043 & 33 \\
\hline 28,1 & 207 & 60 & 0,30 & 34,41 & 0,000 & 2,034 & 1,73 & 0,193 & 1,26 & 0,73 & 0,076 & 1,18 & 1140 & 13 & 1127 & 19 & 1102 & 24 & -3 & 1131 & 23 \\
\hline 29,1 & 250 & 144 & 0,60 & 22,76 & 0,462 & 0,912 & 2,48 & 0,106 & 1,21 & 0,49 & 0,063 & 2,17 & 647 & 7 & 658 & 16 & 696 & 46 & 7 & 648 & 15 \\
\hline 30,1 & 404 & 188 & 0,48 & 48,73 & 0,396 & 1,260 & 2,25 & 0,140 & 1,15 & 0,51 & 0,065 & 1,93 & 844 & 9 & 828 & 19 & 785 & 41 & -8 & 841 & 18 \\
\hline 31,1 & 473 & 491 & 1,07 & 127,22 & 0,769 & 4,988 & 1,36 & 0,311 & 1,10 & 0,81 & 0,116 & 0,80 & 1743 & 17 & 1817 & 25 & 1903 & 14 & 8 & 1903 & 29 \\
\hline 33,1 & 319 & 475 & 1,54 & 29,21 & 0,172 & 0,905 & 1,91 & 0,106 & 1,16 & 0,61 & 0,062 & 1,52 & 652 & 7 & 654 & 13 & 663 & 33 & 2 & 652 & 14 \\
\hline 35,1 & 72 & 44 & 0,63 & 6,45 & 0,241 & 0,891 & 3,42 & 0,104 & 1,71 & 0,50 & 0,062 & 2,97 & 636 & 10 & 647 & 22 & 687 & 63 & 7 & 637 & 20 \\
\hline 36,1 & 299 & 78 & 0,27 & 41,02 & 0,045 & 1,599 & 1,54 & 0,159 & 1,19 & 0,78 & 0,073 & 0,97 & 954 & 11 & 970 & 15 & 1006 & 20 & 5 & 1006 & 39 \\
\hline 38,1 & 3032 & 1745 & 0,59 & 297,70 & 0,506 & 0,967 & 1,33 & 0,114 & 1,04 & 0,78 & 0,062 & 0,83 & 694 & 7 & 687 & 0 & 663 & 18 & -5 & 690 & 13 \\
\hline 40,1 & 311 & 122 & 0,40 & 63,77 & 0,057 & 3,112 & 1,29 & 0,238 & 1,13 & 0,88 & 0,095 & 0,61 & 1378 & 14 & 1436 & 18 & 1522 & 12 & 9 & 1522 & 23 \\
\hline 41,1 & 588 & 315 & 0,55 & 79,40 & 0,667 & 1,451 & 1,87 & 0,156 & 1,10 & 0,59 & 0,067 & 1,52 & 935 & 10 & 910 & 17 & 850 & 31 & -10 & 850 & 62 \\
\hline 42,1 & 411 & 216 & 0,54 & 54,72 & 0,094 & 1,483 & 1,45 & 0,155 & 1,11 & 0,77 & 0,069 & 0,93 & 928 & 10 & 924 & 13 & 912 & 19 & -2 & 925 & 17 \\
\hline
\end{tabular}

\section{DISCUSSIONS}

\subsection{Espinhaço Supergroup}

The occurrence of Espinhaço Supergroup rocks within the cratonic area is related to a branch of the Espinhaço rift system, named Pirapora Aulacogen (Alkmim \& Martins-Neto 2001, Alkmim 2004, Alkmim 2011). The identification of such structure is supported by (i) low values of Bouguer anomaly, showing a NW-trending valley crossing the whole craton (Reis 2011); (ii) remarkable thickening of the paleo/mesoproterozoic units, showed by regional seismic profiles (Coelho et al. 2008, Zalán \& Romeiro-Silva 2007, Hercos et al. 2008); (iii) recently reported transversal seismic profiles showing a deep depression controlled by large scale normal faults (Reis et al. 2013).

The Pirapora Aulacogen is limited by two basement highs, named Januária (NE) and Sete Lagoas (SW), which are remarkable features in the Bouger Anomaly maps (Reis 2011). These structures may have exerted a major control in the filling of the basin, and possibly have acted as source-areas for the sediments. The Sete Lagoas High crops out in the southernmost portion of the São Francisco Craton, showing Archean TTG complexes and greenstonebelt sequences, and Palaeproterozoic granitoids and metasedimentary sequences (Romano et al. 2013, Teixeira et al. 2000, Alkmim 2004). In turn, the Januária High only crops out near to the homonymous city, showing a few exposures of ortogneisses, granites and diorites (Almeida and Uchigasaki 2003, Radambrasil 1982). There is no geochronological constraint for these rocks but a RbSr age of c. 1970 Ma reported in Radambrasil (1982). Nevertheless, an Archean age is considered in the Geological Map of Minas Gerais (COMIG, 2003).

Through paleocurrent measurements and petrographic analysis on the Espinhaço Supergroup units, Lopes (2012) proposed a high-K source-area located northeast of the Cabral ridge. This sourcearea could have fed the Espinhaço basin at the Pirapora rift branch, but probably fed also the main rift, since the same zircon populations are found at the Mineira ridge. Thus, the zircons older than 1800 $\mathrm{Ga}$, found in both Galho do Miguel Formation and Conselheiro Mata Group, may have come from the basement of the São Francisco Craton at the Januária High, which would be exposed as a topographic high at the time of deposition. It is also 
considered that other topographic highs with archean/paleoproterozoic rocks could be contributed with sediments (e.g. Sete Lagoas High). For the main rift, at the Espinhaço range, some other basement highs would have acted as source areas (e.g. Porteirinha Block).

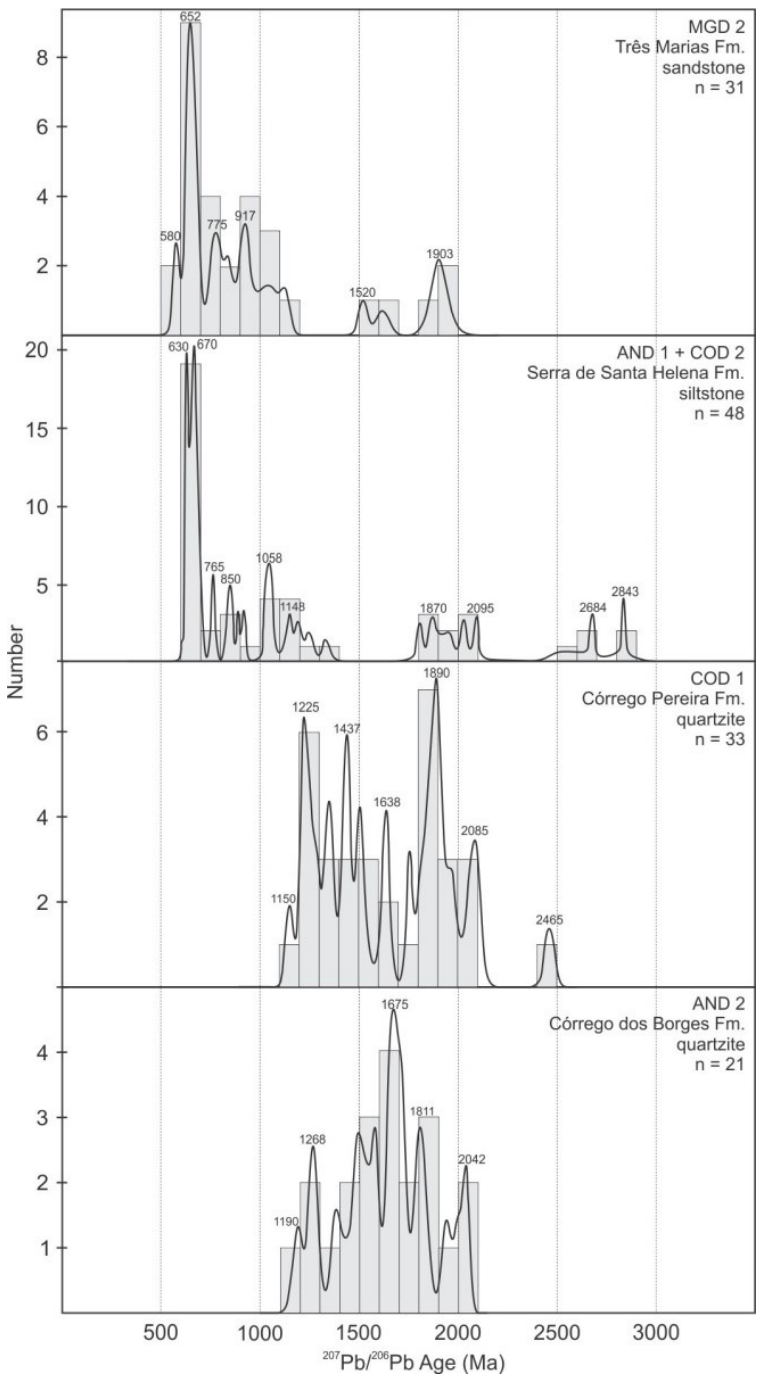

Figure 4. Frequency histogram and probability curves of zircon ages for the analyzed samples.

The zircon populations found in samples of the Galho do Miguel Formation vary substantially. In the Cabral ridge (Lopes 2012) as well as in the southern Espinhaço ridge (Chemale Jr. et al. 2012), the main population is c. 2000-2200 Ma. In southern Cabral ridge, there is a rise in the Archean contribution, as recorded by a significant peak of c. $2700 \mathrm{Ma}$ (PE-SC45, Lopes 2012). To the north of the Cabral ridge, the archean contribution decreases considerably, and arises a new and important population of $c$. $1600 \mathrm{Ma}$. This pattern suggests some differences in the source-area composition, which could be closely related with the litological differences between the Sete Lagoas and Januária highs.
As pointed out by Kuchenbecker (2014), the sedimentary processes which took place in the evolution of the São Francisco Craton show a remarkable polycyclic nature. In this sense, should be considered that an important part of the sediment supply of the Espinhaço Basin may have come from older sedimentary sequences. The Costa Sena Group (Fogaça et al. 1984, Machado et al. 1989), the Riacho dos Machados Group (Crocco et al. 2006) and the Limoeiro Complex (Penha and Sabóia 1995, Knauer 2007), which occur in the Espinhaço ridge, the Minas and Rio das Velhas supergroups, which occur within the craton, are strong candidates. This possibility is illustrated by the great overlap in the detrital zircon age spectra from all those units (Fig. 5).

At Mineira ridge, the archean contribution in the Conselheiro Mata Group seems to be more expressive in the more basal units, decreasing to the top. This relation suggests changes in the source areas during the basin filling, possibly related to the drowning of the basement highs. In fact, rocks correlated to the upper Espinhaço units directly covering the basement highs have been found in several places, in drillcores (Reis et al. 2013) and outcrops (Costa et al. 2015), indicating that this unit has covered a wide portion of the craton. This assumption is also confirmed by seismic interpretation (e.g. Reis et al. 2013).

The zircon population of c.1750 Ma, found at the study area in samples from Córrego dos Borges, Córrego Pereira and Galho do Miguel formations may come from the Statherian magmatism related to the opening of the Espinhaço Basin, as previously proposed by Lopes (2012). However, the large gap between this magmatic event and the deposition of the Conselheiro Mata Group units, constrained here in c. $1200 \mathrm{Ma}$, brings up a complex scenario. If the deposition of the Espinhaço Supergroup took place in a continuous evolution (as proposed in some works, e.g. Dussin \& Dussin 1995, Martins-Neto 2009, Knauer 2007), at $1200 \mathrm{Ma}$ the such magmatic rocks should be buried under a significant sediment pile, and unlikely would be available to generate sediment. On the other hand, if the Espinhaço Supergroup has evolved in a discontinuous basis, with a significant hiatus between its units (as proposed by some authors, e.g. Chemale Jr. et al. 2012, Alkmim and Martins-Neto 2011), the question could be answered by the erosional phase between the two Espinhaço $1^{\text {st }}$ order sequences. 


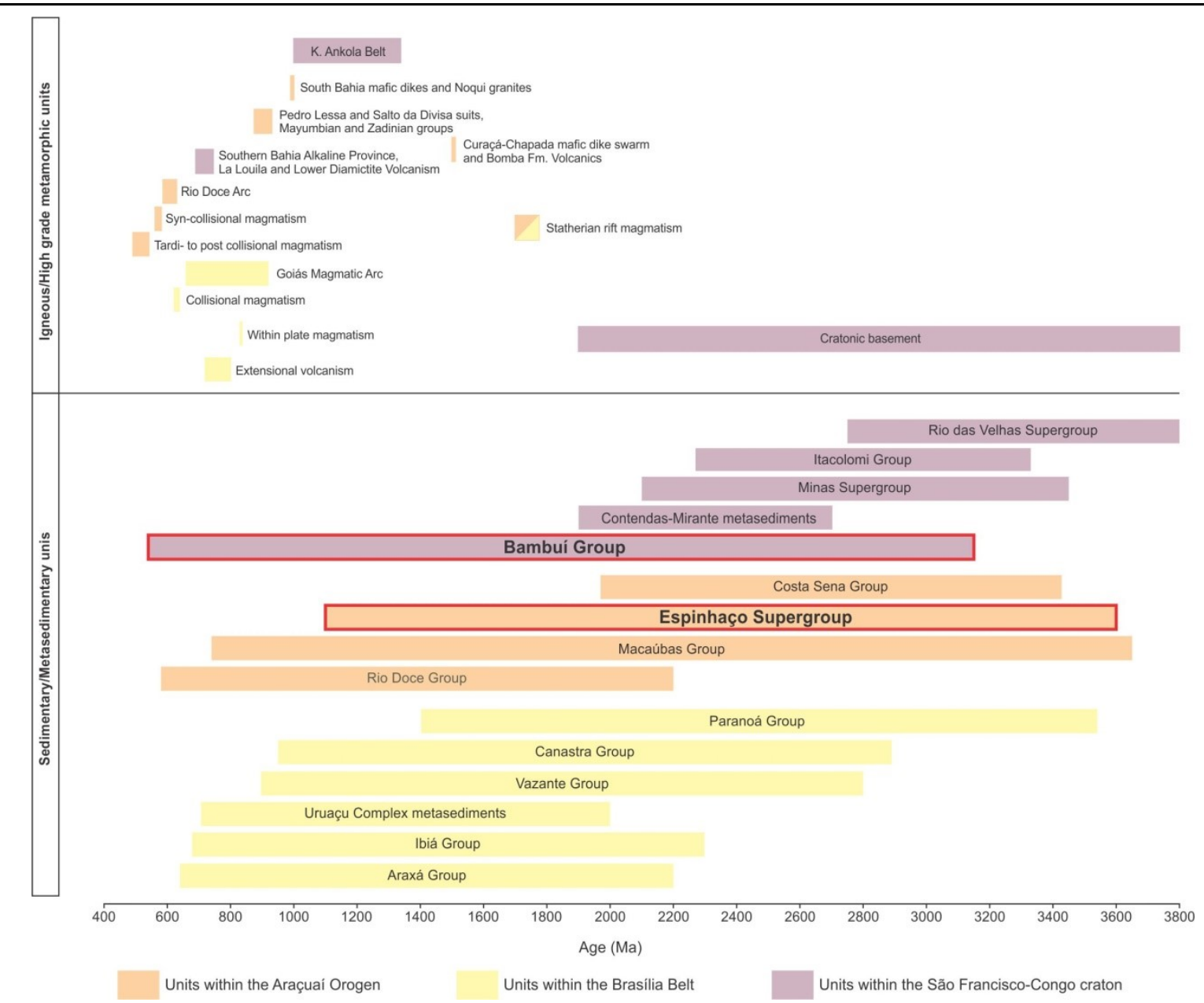

Figure 5. Provenance sketch illustrating the time spam (for igneous and high grade metamorphic rocks) and detrital zircon ages range (for sedimentary and metasedimentary rocks) of several units in the context of the São Francisco Craton. The studied units are highlighted in red. Source: Kuchenbecker (2014) and references therein.

The new data constrain considerably the depositional age of the Conselheiro Mata Group. The zircons aging c. $1200 \mathrm{Ma}$, recovered from Córrego dos Borges and Córrego Pereira formations are the youngest zircons reported by the units and could represent its maximum depositional age. However, the small amount of zircons with such ages requires a more conservative interpretation. The mean age of the youngest representative population of the Córrego dos Borges Formation is $1282 \pm 24 \mathrm{Ma}$, and we assume it as the maximum depositional age of the formation and units above it. Since the whole Conselheiro Mata Group is intruded by mafic dykes of the Pedro Lessa Metaigneous Suíte dated in $933 \pm 20 \mathrm{Ma}$ (Dussin and Chemale, 2012), the deposition of the group at the study area is constrained between c. $1280 \mathrm{Ma}$ and $933 \mathrm{Ma}$.

\subsection{Bambuí Group}

The geotectonic framework concerning the origin and evolution of Bambuí Basin is very complex, since it lies between two orogenic belts with diachronic evolutionary histories. The link between the early stages of the Bambuí Basin and the uplift of the Brasília Belt is now almost consensual. Evidences of such relation lies on (i) the geometry of the deposits, which define an estward-tapering wedge, ranging from c. $3 \mathrm{~km}$ thick near to the Brasília Belt to a few hundred meters close to the Araçuaí Orogen, as indicated by regional seismic profiles and borehole data (Reis et al. 2013, Romeiro-Silva, 1997); (ii) low values of Bouguer anomaly adjacent and parallel to the Brasília belt, defining a $\mathrm{N}$-trending fosse (Reis 2011, Alkmim 2004); (iii) the tectonic imbrication between older rocks from the Brasília belt and locally-derived rocks from the Bambuí Group (Valeriano 1992, Castro 1997, Martins-Neto et al. 2001); (iv) the regional distribution of depositional systems, including conglomeratic units deposited by gravitational flows fed by the Brasília belt orogenic front (Samburá and Lagoa Formosa formations Uhlein et al. 2011, Castro e Dardenne 2000); (v) provenance studies indicating source-areas in the Brasília Belt (Kuchenbecker et al. 2013, Castro and Dardenne 2000, Guimarães and Dardenne 1998).

The relation of the Bambuí Basin with the Araçuaí Orogen is not so clear. The Bambuí Group rocks are affected by the regional deformation related to the collisional stages of the orogen (c. 
580-530 Ma, Pedrosa Soares et al. 2007). If the Araçuaí uplift occurred concurrent to sedimentation, the crustal load and the availability of new sourceareas to the east may have greatly influenced the basing filling. According to Martins-Neto and Alkmim (2001), only the topmost units of the Bambuí Group received sediments from the Araçuaí Orogen, which is corroborated by the occurrence of transitional to continental deposits at the eastern border of the basin, related to its clogging stage (Gorutuba Fm. Kuchenbecker 2014, Costa 2011, Iglesias and Uhlein 2008, Chiavegatto et al. 1997). However, the presence of zircons as young as $550 \mathrm{Ma}$ (PaulaSantos et al. 2015, Pimentel et al. 2012), as well as the Ediacaran guide fossil Cloudina sp. (Warren et al. 2014), both found in the Sete Lagoas Formation, suggest that the entire Bambui Group could have received sediment from the Araçuaí Orogen.

The analyses performed in the Serra de Santa Helena Formation are the first ones reported to the eastern portion of the Bambuí Basin. The zircons aging c. $630 \mathrm{Ma}$ are among the youngest ones reported by the formation throughout the basin, and this is interpreted as its maximum depositional age at the study area. Potential source rocks with this age occur in both Araçuaí Orogen and Brasília Belt. In the Araçuaí Orogen, the Rio Doce magmatic arc presents plutonic rocks from $630 \mathrm{Ma}$ to $585 \mathrm{Ma}$ (Pedrosa-Soares et al. 2007), while in the Brasília Belt arc magmatism (Pimentel et al. 1999), collisional magmatism (Seer and Moraes 2013) and metamorphism are recognized in c. $630 \mathrm{Ma}$. Due to the pelagic nature of the Serra de Santa Helena Formation rocks, is difficult to decide between these two possible sources from paleocurrent measurements.

For the older age clusters observed in the Serra de Santa Helena Formation (760-900 Ma, 1000 1200Ma, 1800-2100 Ma and 2600-2800 Ma) should be considered the potential primary and secondary sources. In both Brasília Belt and Araçuaí Orogen thare are several sedimentary units which contain zircons of the same age of those found in the Bambuí Group (Fig. 5). In this sense, the Canastra, Araxá and Paranoá Groups, from the Brasília Belt side, and the Macaúbas Group and the Espinhaço Supergroup, in the Araçuaí Orogen, are strong candidates.

Primary Archean sources are available in the Brasília Belt (Goiás Massif and other basement inliers) and in the Araçuaí Orogen (Gouveia, Porteirinha and Guanhães complexes), but also in the São Francisco Craton basement. Since the Bambuí Group lies directly on the cristaline basement in several places, it seems to be plausible to assume that the basement highs (Sete Lagoas and Januária) have acted again as source areas. They may have been reactivated by positive epirogeny, in response to the litospheric load of Brasília Belt, to the west, setting the forebulge of the Bambui foreland basin.

The results obtained for the Três Marias Formation sandstone could contribute to understand the Bambuí Basin evolution in the studied area. The zircons with ages older than 630 Ma have potential source areas in both Brasília Belt and Araçuaí Orogen, as discussed above. However, the presence of zircons as young as $580 \mathrm{Ma}$ is very relevant, and strongly suggests that the Araçuai Orogen had contributed as a source area. Rocks with this age are widespread distributed in the Araçuaí Orogen, mainly related to magmatism in the syncollisional stage (Fig. 5).

\section{CONCLUSIONS}

The $\mathrm{U}-\mathrm{Pb}$ results here presented were interpreted together with the available data, in order to contribute to the age constraints for deposition and sedimentary provenance study of the Espinhaço Supergroup and Bambuí Group within the São Francisco Craton and its borders. The analysis of all data allows us to list some conclusions:

I. An important part of the sediment supply for the Espinhaço and Bambuí basins in the studied area could have come from older sedimentary units, as suggested by the great overlap in their detrital zircon age spectra. It demonstrates the remarkable polycyclic nature of the sedimentary processes who took place in the São Francisco Craton evolution;

II. The cratonic basement highs (Sete Lagoas and Januária) seem to have been important source areas to the Mesoproterozoic units of the upper Espinhaço Supergroup, feeding the Pirapora Aulacogen. Lithologic differences between the two highs are recorded in the adjoining sedimentary deposits within the aulacogen. Such structures may have also acted as topographic highs during the deposition of the Bambuí Group, after being reactivated by positive epirogeny, in response to the litospheric load of Brasília Belt;

III. Within the São Francisco Craton and at the Mineira ridge, the deposition of the Conselheiro Mata Group could be constrained between 1280 Ma (younger zircon population) and $933 \mathrm{Ma}$ (age of intrusive basic rocks);

IV. The age spectrum of the Serra de Santa Helena Formation zircons suggests that its sediments may have come both from the Brasilia Belt and the Araçuaí Belt. On the other hand, zircons as young as $580 \mathrm{Ma}$, found in the Três Marias 
Formation sandstones suggest contribution from the Araçuaí Orogen.

\section{ACKNOWLEDGMENTS}

The authors acknowledge financial support provided by the Brazilian Geological Survey - CPRM and the staff of the geochronology laboratory of the Australian National University.

\section{REFERENCES}

Almeida, F.F.M. 1977. O Cráton do São Francisco. Revista Brasileira de Geociências 7, 349-364.

Almeida, A.M.,Uchigasaki, K. 2003. Mapeamento geológico em área dos municípios de Cônego Marinho e Januária -MG. Graduate Work. UFMG, $83 p$.

Almeida-Abreu, P.A. 1995. O Supergrupo Espinhaço da Serra do Espinhaço Meridional Minas Gerais : o rifte, a bacia e o orógeno. Geonomos 3 (1), 1-18.

Almeida-Abreu, P.A., Queiroz, W.P., Rosière, C.A., Renger, F.E. 2001. A bacia foredeep do Orógeno Espinhaço da Serra do Espinhaço Meridional em Minas Gerais. Proceedings of the VIII SNET, Recife, Brazil.

Alkmim, F.F. 2004. O que faz de um cráton um cráton? O Cráton do São Francisco e as revelações Almeidianas ao delimitá-lo. In: Almeida, F. F. M. \& Mantesso-Neto, V. (eds.). Geologia do Continente Sul-Americano: evolução da obra de Fernando Flávio Marques de Almeida. Beca Produções Culturais, São Paulo, 17-35

Alkmim, F.F. 2011. Geologia e Tectônica da Porção Sudoeste da Bacia do São Francisco. In: Pedrosa-Soares A. C., Noce C. M. Voll E., Kuchenbecker M., Reis H. L. S., Fragoso D. G. C. (Eds) Projeto Alto Paranaíba. Belo Horizonte, CODEMIG, 10-67.

Alkmim, F.F.; Martins-Neto, M.A. 2001. A Bacia Intracratônica do São Francisco: Arcabouço Estrutural e Cenários Evolutivos. In: Pinto, C.P.; Martins-Neto, M.A. 2001. Bacia do São Francisco: Geologia e Recursos Naturais. SBG, Belo Horizonte, 9-30.

Alkmim, F.F., Marshak, S., Pedrosa-Soares, A.C., Peres, G.G., Cruz S.C., Whittington, A., 2006. Kinematic evolution of the Araçuaí-West Congo orogen in Brazil and Africa: nutcracker tectonics during the Neoproterozoic assembly of Gondwana. Precambrian Research 149, 43-63.

Babinski, M., Vieira, L.C., Trindade, R.I.F., 2007. Direct dating of the Sete Lagoas cap carbonate (Bambuí Group, Brazil) and implications for the Neoproterozoic glacial events. Terra Nova 19, 401-406.

Black, L.P., Kamo, S.L., Allen, C.M., Aleinikoff, J.N., Davis, D.W. Korsch, R.J., Foudoulis, C. 2003 TEMORA 1: a new zircon standard for Phanerozoic U-Pb geochronology. Chemical Geology 200, 780 155-170

Castro, P.T.A.,Dardenne, M.A. 2000.The sedimentology,stratigraphy and tectonic context of the São Francisco Supergroup at the southern boundary of the São Francisco craton, Brazil. Revista Brasileira de Geociências 30, 345-437.

Caxito, F.A., Halverson, G.P., Uhlein, A., Stevensson, R., Dias, T.G., Uhlein, G.J., 2012. Marinoan glaciation in east Central Brazil. Precambrian Research 200-203, 38-58.

Chemale Jr., F., Dussin, I.A., Alkmim, F.F., Martins, M.S., Queiroga G., Armstrong, R., Santos, M. N. 2012. Unravelling a Proterozoic basin history through detrital zircon geochronology: the case of the Espinhaço Supergroup, Minas Gerais, Brazil. Gondwana Research, v. 22, n.1, p.200-206.
Chiavegatto J.R.S. 1992. Análise estratigráfica das sequências tempestíticas da Fm. Três Marias (Proterozóico Sup.), na porção meridional da Bacia do São Francisco. Dissertação de Mestrado, Universidade Federal de Ouro Preto, 196 p.

Chievegatto, J.R.S., Gomes, N.S., Dardenne, M.A. 1997. Conglomerados oligomíticos da Formação Três Marias na Serra do Gorutuba, norte de Minas Gerais. 9o Simpósio de Geologia de Minas Gerais. v. 14, 83-84. Ouro Preto: SBG.

Coelho, J.C.C., Martins-Neto, M.A., Marinho, M.S. 2008. Estilos estruturais e evolução tectônica da porção mineira da bacia proterozóica do São Francisco. Revista Brasileira de Geociências 38(2 - suplemento), 149-165.

COMIG - COMPANHIA MINERADORA DE MINAS GERAIS. 2004. Mapa Geológico do Estado de Minas Gerais - 1:1.000.000. Available on http://www.codemig.com.br/uploads/ mapag.pdf. Accessed in 28/9/13

Costa, D. A. 2011. Controle lito-estrutural e estratigráfico na hidrogeoquímica e nas concentrações de fluoreto no sistema aquífero cárstico-fissural do Grupo Bambuí, norte de Minas Gerais. Masters Dissertation, Universidade Federal de Minas Gerais, Belo Horizonte, 138p.

Costa, R.D., Kuchenbecker, M., Chaves, M.L.S.C., Leite, M.M. 2015. Geologia da Porção Mineira das folhas Rio Itaguari e Serra dos Tropeiros 1:100.000, Minas Gerais (texto explicativo, mapa geológico, SIG e mapa de pontos). 1. ed. Belo Horizonte: CODEMIG-Companhia de Desenvolvimento Econômico de Minas Gerais. v. 1. 25p.

Crocco, F.A., Abreu, F.R., Teixeira,N., Cançado, F.L.L., Maciel, J.D., Sena, F., Ramos, M.M. 2006. Mineralizações de Zinco e Chumbo do depósito Salobro, Porteirinha (MG). Geociências 25(3), 359-378.

Dussin, I.A., Dussin, T.M. 1995. Supergrupo Espinhaço: modelo de evolução geodinâmica. Geonomos, 3: 19-26.

Guimarães, E.M., Dardenne, M.A.,1998. Características de coberturas cratônicas relacionadas com faixas de deformação: as bacias Paranoá e Bambuí e a evolução da faixa dobrada Brasília. Proceesings of the XL Brazilian Geological Congress.

Hercos, C.M., Martins-Neto, M., Danderfer Filho, A. 2008. Arcabouço estrutural da Bacia do São Francisco nos arredores da Serra da Água Fria (MG), a partir da integração de dados de superfície e subsuperfície. Revista Brasileira de Geociências 38 (2), 197-212.

Iglesias, M., Uhlein, A. 2008. Estratigrafia do Grupo Bambuí na Serra do Jaíba, norte de Minas Gerais. Geonomos, 16 (1), 2127.

Knauer, L. G. 1999. Serra do Espinhaço Meridional: Considerações sobre a estratigrafia e a análise da deformação das unidades proterozóicas. PhD Thesis, UNESP-Rio Claro, 244p.

Knauer, L.G. 2007. O Supergrupo Espinhaço em Minas Gerais: considerações sobre sua estratigrafia e seu arranjo estrutural. Geonomos, v. 15, nำ. p. 81 - 90 .

Knauer,L.G., Ebert,H.D. 1997. Estruturação tectônica da região de Diamantina, MG e considerações sobre a idade do Orógeno Espinhaço. Proceedings of the VI SNET, 56-58.

Knauer, L.G., Lopes-Silva, L.L., Souza, F.B.B., Silva, L.R., Carmo, R.C. 2007. Nota explicativa da Folha Monte Azul- SD.23-Z-D-II, escala 1:100.000. Convênio UFMG/CPRM.

Kuchenbecker, M., 2011. Quimioestratigrafia e proveniência sedimentar da porção basal do Grupo Bambuí em Arcos (MG). Masters Dissertation, Universidade Federal de Minas Gerais, Belo Horizonte, 91p. 
Kuchenbecker, M., Babinski, M., Pedrosa-Soares, A. C., Costa, R.D., Lopes-Silva, L., Pimenta, F. 2013. Proveniência e análise sedimentar da porção basal do Grupo Bambuí em Arcos (MG). Geol. USP, Sér. cient. 13(4), 4-61.

Kuchenbecker, M. 2014. Relações entre coberturas do Cráton do São Francisco e bacias situadas em orógenos marginais: o registro de datações $\mathrm{U}-\mathrm{Pb}$ de grãos detríticos de zircão e suas implicações geotectônicas. PhD Thesis. Universidade Federal de Minas Gerais, Belo Horizonte, 175p.

Lopes, T.C. 2012. O Supergrupo Espinhaço na Serra do Cabral, Minas Gerais: contribuição ao estudo de proveniência sedimentar. Master's Dissertation. Universidade Federal de Minas Gerais. 136p.

Ludwig, K.R. 2001. User's manual for Isoplot/Ex Version 2.49 A geochronological toolkit for Microsoft Excel. Berkeley Geochronological Center, Special Publication 1a, 55.

Ludwig, K.R. 2003. Using Isoplot/Ex, version 3.00, a geochronological toolkit for Microsoft Excel, Berkeley Geochronology Center Special Publication No. 1. 43p.

Machado N., Schrank A., Abreu F.R., Knauer L.G., Almeida-Abreu P.A. 1989. Resultados preliminares da geocronologia U-Pb na Serra do Espinhaço Meridional. In: Proceedings of the Simpósio de Geologia de Minas Gerais 5, 171-174.

Martins-Neto, M.A. 1998. O Supergrupo Espinhaço em Minas Gerais: registro de uma bacia rifte-sag do Paleo/Mesoproterozóico. Revista Brasileira de Geociências 28(2),151-168

Martins-Neto, M.A. 2009. Sequence stratigraphic framework of Proterozoic successions in eastern Brazil. Marine and Petroleum Geology 26, 163-176.

Martins-Neto, M.A., Alkmim, F.F. 2001. Estratigrafia e evolução tectônica das bacias neoproterozóicas do paleocontinente São Francisco e suas margens: Registro da quebra de Rodínia e colagem de Gondwana. In: C.P. Pinto \& M.A. Martins-Neto (eds.) Bacia do São Francisco: Geologia e Recursos Naturais, SBG/Núcleo MG, 31-54.

Martins-Neto, M.A., Pedrosa-Soares, A.C., Lima, S.A.A. 2001. Tectono-sedimentary evolution of sedimentary basins from Late Paleoproterozoic to Late Neoproterozoic in the São Francisco craton and Araçuaí fold belt, eastern Brazil. Sedimentary Geology 141-142, 343-370.

Paula-Santos, G.M, Babinski, M., Kuchenbecker M., Caetano-Filho, S., Trindade, R.I., Pedrosa-Soares, A.C. 2015. New evidence of an Ediacaran age for the Bambuí Group in southern São Francisco craton (eastern Brazil) from zircon $\mathrm{U}-\mathrm{Pb}$ data and isotope chemostratigraphy. Gondwana Research 28 (2), 702720.

Pedrosa-Soares, A.C., Noce, C.M., Wiedemann, C.M., Pinto, C.P., 2001. The Araçuaí-West Congo orogen in Brazil: an overview of a confined orogen formed during Gondwanland assembly. Precambrian Research 110, 307-323.

Pedrosa-Soares, A. C., Noce, C. M., Alkmim, F. F., Silva, L. C., Babinski, M., Cordani, U., Castañeda, C. 2007. Orógeno Araçuaí: síntese do conhecimento 30 anos após Almeida 1977. Geonomos 15, 1-16.

Penha, U.C., Sabóia L.A. 1995. Dados preliminaries e potencial metalogenético da Sequência Limoeiro, Serra do Espinhaço Setentrional Mineiro. Anais do 8o Simpósio de Geologia de Minas Gerais. Boletim da Sociedade Brasileira de Geologia 13, 32-33.

Pimentel, M.M, Fuck R.A., Botelho, N.F. 1999. Granites and the geodynamic history of the Neoproterozoic Brasilia Belt, Central Brazil: a review. Lithos 46(3), 463-483.
Pimentel, M., Rodrigues, J.B., Della Giustina, M.E.S., Matteini, S.J.M., Armstrong, R., 2011. The tectonic evolution of the Neoproterozoic Brasília Belt, central Brazil, based on SHRIMP and LA-ICPMS U-Pb sedimentary provenance data: A review. Journal of South American Earth Sciences 31, 345-357.

Pimentel, M., Della Giustina, M.E.S., Rodrigues, J.B., Junges, S.L. 2012. Idades dos grupos Araxá e Bambuí: Implicações para a evolução da Faixa Brasília. Proceedings of the 46 Congresso Brasileiro de Geologia, Santos.

Radambrasil. 1982. Geological Map. Folha SD.23 Brasília, 1:1.000.000, Rio de Janeiro.

Reis, H.L.S. 2011. Estratigrafia e tectônica da Bacia do São Francisco na zona de emanações de gás natural do baixo Rio Indaiá (MG). Master's Dissertation. Universidade Federal de Ouro Preto. 156p.

Reis, H.L.S., Costa, R.D., Prezotti, F.P.S., Tedeschi, M., Fonseca, H.A.M., Kuchenbecker, M. 2012. Geologia e recursos minerais da Folha Andrequicé, 1:100.000. CPRM/UFMG.

Reis, H. L. S., Fonseca, R. C. S., Alkmim, F. F., Nascimento, T. C., Suss, J. 2013. A Bacia do São Francisco (MG): registro de uma longa história de ativações e reativações em domínio cratônico. In: 170 Simpósio de Geologia de Minas Gerais e 13은 Simpósio de Geologia do Sudeste, 2013, Juiz de Fora (MG), Brasil.

Rocha-Campos, A.C., Hasui, Y. 1981. Tillites of the Macaúbas Group (Proterozoic) in central Minas Gerais and southern Bahia, Brazil. In: Hambrey, M.J., Harland, W.B. (Eds). Earth pre-pleistocene glacial record. Cambridge University Press, 933-938 p.

Rodrigues, J.B., 2008. Proveniência de sedimentos dos grupos Canastra, Ibiá, Vazante e Bambuí - Um estudo de zircões detríticos e Idades Modelo Sm-Nd. PhD Thesis, Instituto de Geociências, Universidade de Brasília, Brazil.

Rolim, V. K. 1992. Uma Interpretação das Estruturas Tectônicas do Supergrupo Espinhaço, Baseada na Geometria dos Falhamentos de Empurrão. Revista da Escola de Minas 45 (1 e 2), $75-77$.

Romano, R., Lana, C., Alkmim, F.F., Stevens, G., Armstrong, R. 2013. Stabilization of the southern portion of the São Francisco craton, SE Brazil, through a long-lived period of potassic magmatism. Precambrian Research 224, 143- 159.

Romeiro Silva P.C. 1997. A passagem do Mesoproterozóico para o Neoproterozóico no centro-leste do Brasil e o estilo estrutural envolvido. Boletim da Sociedade Brasileira de Geologia, Núcleo MG. no 14, p. 9.

Schöll,W.U., Fogaça, A.C.C. 1979. Estratigrafia da Serra do Espinhaço na região de Diamantina. Proceedings of the 1st Simp. Geol. Minas Gerais, 55-73.

Seer, H., Moraes, L.C. 2013. Within plate, arc, and collisional Neoproterozoic granitic magmatism in the Araxá Group, Southern Brasília belt, Minas Gerais, Brazil. Brazilian Journal of Geology 43(2), 333-354.

Sial, A.N., Dardenne, M.A., Misi, A., Pedreira, A.J., Gaucher, C., Ferreira, V.P., Silva Filho, M.A., Uhlein, A., Pedrosa-Soares, A.C., Santos, R.V., Egydio-Silva, M., Babinski, M., Alvarenga, C.J.S., Fairchild,T.R., Pimentel, M.M. 2009. The São Francisco Palaeocontinent. In: Gaucher, C., Sial, A.N.,Halverson, G.P., Frimmel, H.E. (Eds): Neoproterozoic-Cambrian Tectonics, Global Change and Evolution: a focus on southwestern Gondwana. Developments in Precambrian Geology, 16, Elsevier, pp. 31269.

Silva, L.C., Armstrong, R., Noce, C.M., Carneiro, M.A., Pimentel, M., Pedrosa-Soares, A.C., Leite, C.A., Vieira, V.S., Silva, M.A., Paes, V.J.C., Cardoso Filho, J.M. 2002. Reavaliação da 
evolução geológica em terrenos Pré-cambrianos brasileiros com base em novos dados U-Pb SHRIMP, parte II: Orógeno Araçuaí, Cinturão Mineiro e Cráton São Francisco meridional. Revista Brasileira de Geociências 32(4), 513-528.

Tedeschi, M. F. 2013. Caracterização do Arco Magmático do Orógeno Araçuaí entre Frei Inocêncio e Itambacuri, MG. Masters Dissertation. Universidade Federal de Minas Gerais. $162 p$.

Teixeira W., Sabaté P., Barbosa J., Noce C. M., Carneiro M. A 2000. Archean and Paleoproterozoic tectonic evolution of the São Francisco Craton. In: U.G. Cordani, E.J. Milani, A. Thomaz Filho, D.A. Campos, D. A. (eds.) Tectonic Evolution of South America. Rio de Janeiro, p. 101-138.

Trompette, R. 1994. Geology of Western Gondwana (2000-500 Ma). Pan-African-Brasiliano aggregation of South America and Africa. Rotterdam, A.A. Balkema, $350 \mathrm{p}$.

Uhlein, A., Baptista, M.C., Seer, H.J., Caxito, F.A., Uhlein, G.J., Dardenne, M.A. 2011. A Formação Lagoa Formosa, Grupo Bambuí (MG): Sistema Deposicional de Leque Submarino em Bacia de Ante-País. Geonomos 19(2), 163-172
Vieira, L.C., Trindade, R.I.F, Nogueira, A.C.R., Ader, M., 2007. Identification of a Sturtian cap carbonate in the Neoproterozoic Sete Lagoas carbonate plataform, Bambuí Group, Brazil. C. R. Geoscience 339, 240-258.

Williams I.S. 1998. U-Th-Pb Geochronology by Ion Microprobe. In: M.A McKibben, W.C Sahnks III, W.I Ridley (eds) Applications of microanalytical techniques to understanding mineralizing processes. Reviews in Economic Geology, 7.

Williams I.S. and Meyer C. 1998. U-Pb geochronology of zircons from lunar breccia 73217 using a sensitive high massresolution ion microprobe. Journal of Geophysics Research, 89:B525-B534.

Zalán, P. V. \& Romeiro-Silva, P. C. 2007. Bacia do São Francisco. Boletim de Geociências Petrobrás 15(2): 561-571.

Warren, L.V., Quaglio, F., Riccomini, C., Simões, M.G., Poiré, D.G., Strikis, N.M., Anelli, L.E., Strikis, P.C. (2014). The puzzle assembled: Ediacaran guide fossil Cloudina reveals an old proto- Gondwana seaway. Geology, DOI: 10.1130/G35304.1 